OPEN ACCESS

Edited by:

Julian P. Meeks,

University of Texas Southwestern Medical Center, United States

Reviewed by:

Shin Nagayama,

Texas Medical Center, United States David Henry Gire, University of Washington,

United States

*Correspondence:

Shaolin Liu

Shaolin.liu@howard.edu

Specialty section:

This article was submitted to

Cellular Neurophysiology,

a section of the journal

Frontiers in Cellular Neuroscience

Received: 15 April 2020

Accepted: 20 May 2020

Published: 12 June 2020

Citation:

Liu S (2020) Dopaminergic Modulation of Glomerular Circuits

in the Mouse Olfactory Bulb.

Front. Cell. Neurosci. 14:172. doi: 10.3389/fncel.2020.00172

\section{Dopaminergic Modulation of Glomerular Circuits in the Mouse Olfactory Bulb}

\author{
Shaolin Liu* \\ Department of Anatomy, Howard University College of Medicine, Washington, DC, United States
}

Dopaminergic neurons are located in several brain areas including the olfactory bulb $(\mathrm{OB})$ and involved in many physiological and pathophysiological processes. In the $\mathrm{OB}$, dopamine (DA) is released exclusively by a population of interneurons termed short axon cells (SACs) in the glomerular layer, the initial synaptic integration site of the whole olfactory system. SACs corelease GABA and extend their processes to many glomeruli forming the interglomerular circuit. Two major groups of DA receptors D1like (D1LRs) and D2-like (D2LRs) types are differentially distributed in the OB, i.e., D1LRs are broadly present except the most superficial olfactory nerve (ON) layer while D2LRs are predominantly confined to the ON and glomerular layers, suggesting that they mediate different physiological functions. In contrast to the well-known D2LR-mediated presynaptic inhibition of ON terminals in the $\mathrm{OB}$, the cellular and circuit targets of the D1LR-mediated DA actions remain unclear even though D1LR activation improves odor detection and discrimination. We recently demonstrated that endogenous DA released from SACs or exogenous DA excites a population of excitatory glomerular neurons termed external tufted cells (ETCs) via D1LRs. But the physiological significance of this D1LR activation is largely unknown. In the present study, we addressed these questions by a systematic examination of exogenous DA actions on synaptic activities and excitabilities in most glomerular neurons and $\mathrm{OB}$ output neurons with the following major findings: (1) DA via D1LRs enhances OB output by potentiating the ETC-mediated feedforward excitation to the OB output neurons but suppresses spontaneous excitatory synaptic activities in both types of inhibitory glomerular interneurons periglomerular (PGCs) and SACs; (2) this suppression of excitatory synaptic activities in PGCs and SACs depends on activation of GABA receptors; (3) DA via D1LRs augments spontaneous inhibitory synaptic activities in all glomerular neurons and OB output neurons; (4) DA selectively activates SACs via D1LRs. These findings suggest that activation of D1LRs elevates the system's sensitivity to odor stimuli and provide a mechanistic basis for the functional roles of DA in modulating odor detection and discrimination.

Keywords: dopamine, synaptic transmission, interneuron, olfactory bulb, excitation, inhibition 


\section{INTRODUCTION}

Dopamine (DA) as a neurotransmitter or neuromodulator plays important roles in many brain functions including motor control, motivation, reward, cognition, maternal and reproductive behaviors (Bjorklund and Dunnett, 2007a; Klein et al., 2019). DA actions are mediated by G protein-coupled receptors, which are classified into two major types based on their gene sequence homology and functions: the excitatory D1like (D1LRs) and inhibitory D2-like (D2LRs) receptors (Beaulieu and Gainetdinov, 2011). In the mammalian brain DA-containing neurons are located in several distinctive cell groups distributed from the mesencephalon to the olfactory bulb (OB) (Dahlstroem and Fuxe, 1964; Bjorklund and Dunnett, 2007b).

In the $\mathrm{OB}, \mathrm{DA}$ is exclusively released by a population of interneurons termed short axon cells (SACs), which express glutamic acid decarboxylase (GAD) 67, a key enzyme for biosynthesis of the inhibitory neurotransmitter GABA (Kosaka and Kosaka, 2008; Kiyokage et al., 2010). Both D1LRs and D2LRs are present in the OB with distinct distribution patterns, i.e., D1LRs are broadly present from glomerular to granule cell layers while D2LRs are confined only to the superficial olfactory nerve $(\mathrm{ON})$ and the glomerular layers (Guthrie et al., 1991; Levey et al., 1993; Coronas et al., 1997; Gutierrez-Mecinas et al., 2005; Yu et al., 2019). Pharmacological activation of D2LRs (Nickell et al., 1991; Hsia et al., 1999; Ennis et al., 2001; Maher and Westbrook, 2008; McGann, 2013) or optogenetic activation of SACs inhibits glutamate release from ON terminals to postsynaptic cellular targets in the glomerular layer (Vaaga et al., 2017). Moreover, in vivo studies showed that blocking either D1LRs or D2LRs (Escanilla et al., 2009) or lack of DA transporters or D2LRs impairs odor discrimination in animals (Tillerson et al., 2006; Taylor et al., 2009) whereas olfactory deprivation upregulates D2LR density in the OB (Guthrie et al., 1991). Interestingly, a mating-triggered DA surge in the main $\mathrm{OB}$ of female mice impairs their perception of social odors contained in male urine (Serguera et al., 2008). Additionally, dopaminergic SACs exhibit high level of activitydependent plasticity. For example, naris closure leads to a drastic reduction in the number of $\mathrm{TH}$-immunoreactive cells and processes (Cave and Baker, 2009; Parrish-Aungst et al., 2011; Lazarini et al., 2014; Bonzano et al., 2016). Furthermore, majority of DAergic SACs originate from postnatal neurogenesis and are continuously subject to replenishment during adult life (Lazarini et al., 2014; Bonzano et al., 2016). All these previous findings suggest that DA actively participates in modulating signal processing in the $\mathrm{OB}$. However, the mechanistic actions of DA at the cellular and circuit levels in the $\mathrm{OB}$ are not well understood.

The DAergic/GABAergic SACs have somata and processes confined to the glomerular layer (Kosaka and Kosaka, 2008; Kiyokage et al., 2010), where excitatory and inhibitory circuits are formed among the axonal terminals of olfactory sensory neurons (OSNs), apical dendrites of ETCs and the OB output neurons mitral and tufted cells (MTCs), and two major populations of inhibitory interneurons types - SACs and periglomerular cells (PGCs) (Lledo et al., 2005; Wilson and Mainen, 2006;
Nagayama et al., 2014; Burton, 2017; Pignatelli and Belluzzi, 2017). Thus, the DAergic/GABAergic SACs are well-situated to modulate olfactory signal processing at the initial site of synaptic integration in the whole olfactory system. Physiological evidence suggests that functional operation of the SACderived circuit depends on postsynaptic cellular targets. For instance, DA is coreleased with GABA from SACs to mediate a biphasic excitatory-inhibitory sequential response in ETCs (Liu et al., 2013) while the transmission from SACs to MTCs or PGCs is mediated by GABAergic and electrical synapses (Banerjee et al., 2015; Liu et al., 2016) or GABA alone but not by DA (Shao et al., 2019). Alternatively, DA potentially functions as a neuromodulator in the $\mathrm{OB}$ through volume transmission (Cragg et al., 2001). A recent study revealed that DA release from SACs in the $\mathrm{OB}$ was quantal and calcium-dependent but was asynchronous and lasted for tens of seconds (Borisovska et al., 2013), supporting volume transmission. Thus, in the present study we designed experiments to examine actions of exogenous DA on both excitatory and inhibitory transmission in the glomerular circuit and analyzed the underlying cellular mechanisms.

\section{MATERIALS AND METHODS}

\section{Animals}

Both male and female C57BL/6J mice were purchased from Charles River. The GAD2gfp mice provided of courtesy by Dr. Gabor Szabo were initially from the GAD65_3e/gfp5.5 \#30 line on a genetic background of C57BL6 backcrossed to B6CBAF1/J wild-type mice to generate the transgene heterozygotes (LopezBendito et al., 2004). TH-GFP mice were initially courtesy of Dr. Kobayashi (Matsushita et al., 2002) and generated using a $9.0 \mathrm{~kb} 5^{\prime}$-flanking region of the $\mathrm{TH}$ gene on a genetic background of C57BL/6J to crossbreed with DBA/2J mice. These TH-GFP transgenic mice were then backcrossed to $\mathrm{C} 57 \mathrm{BL} / 6 \mathrm{~J}$ mice to generate heterozygotes for experimental usage. All animals were maintained with a standard 12-h light/dark cycle with ad libitum access to food and water. All experimental procedures were carried out in accordance with protocols submitted to and approved by the Howard University Institutional Animal Care and Use Committee.

\section{Slice Preparation}

Acute OB slices were prepared from 6- to 8-week-old male or female mice as described previously (Liu et al., 2013). Briefly, horizontal slices $(350 \mu \mathrm{m})$ were cut with a VT1200s vibratome (Nussloch, Germany) in an ice-cold and oxygenated $\left(95 \% \mathrm{O}_{2}-\right.$ $5 \% \mathrm{CO}_{2}$ ) sucrose-based artificial CSF (sucrose-ACSF) containing (in mM) 210 sucrose, $3 \mathrm{KCl}, 1.2 \mathrm{NaH}_{2} \mathrm{PO}_{4}, 2.6 \mathrm{MgSO}_{4}, 0.5$ $\mathrm{CaCl}_{2}, 26 \mathrm{NaHCO}_{3}, 10$ glucose. After $30 \mathrm{~min}$ incubation in normal ACSF at $30^{\circ} \mathrm{C}$, slices were then transferred to ACSF at room temperature until they were used for recordings. Normal ACSF was continuously bubbled with $95 \% \mathrm{O}_{2}-5 \% \mathrm{CO}_{2}$ and had the following composition (in $\mathrm{mM}$ ): $124 \mathrm{NaCl}, 3 \mathrm{KCl}, 1.25$ $\mathrm{NaH}_{2} \mathrm{PO}_{4}, 2.0 \mathrm{MgSO}_{4}, 2.0 \mathrm{CaCl}_{2}, 26 \mathrm{NaHCO}_{3}, 10$ glucose. 
During experiments, slices were perfused at $3 \mathrm{ml} / \mathrm{min}$ with ACSF equilibrated with $95 \% \mathrm{O}_{2}-5 \% \mathrm{CO}_{2}$ and warmed to $30^{\circ} \mathrm{C}$.

\section{Electrophysiology}

Whole cell patch clamp recordings were made from $\mathrm{OB}$ neurons visualized using Axio Examiner (Zeiss, Oberkochen, Germany) fixed-stage upright microscope with near-infrared differential interference contrast (DIC) optics. Short axon (SACs) and periglomerular cells (PGCs) were identified by their expression of TH- or GAD65-EGFP in corresponding transgenic mice, respectively.

External tufted cells (ETCs) were initially reported in previous studies with Golgi staining (Romon and Cajal, 1909; Pinching and Powell, 1971a,b; Macrides and Schneider, 1982). Recent work more rigorously characterized the morphological and electrophysiological properties of ETCs (Hayar et al., 2004b; Antal et al., 2006). Based on findings from these studies, we redefined and identified ETCs with the following criteria: (1) spontaneous and intrinsic burst spiking that is resistant to blockers of fast synaptic transmitter receptors; (2) soma residing in the deep half of the glomerular layer with "pear" appearance viewed in near-IR DIC optics; (3) an apical dendrite tuft extensively ramifying in a single glomerulus but lack of lateral dendrites in the external plexiform layer (EPL).

To localize apical dendrite tufts of the recorded mitral/tufted cells (MTCs) in the glomerular layer, Alexa-594 (10 $\mu \mathrm{M})$ was added to the recording electrode solution. Whole cell current or voltage signals were recorded with a MultiClamp 700B amplifier (Molecular Devices, Palo Alto, CA, United States) and low-pass filtered at $4 \mathrm{kHz}$ and sampled at $10 \mathrm{kHz}$ with a DIGIDATA 1550B 16-bit analog-to-digital converter (Molecular Devices) using Clampex 11.0.3 (Molecular Devices). Patch recording electrodes were pulled from standard-wall glass capillary tubes without filament (Sutter Instrument, Novato, CA, United States). Patch pipettes (4-7 M $\Omega$ ) for whole cell current clamp recording contained (in $\mathrm{mM}$ ) $115 \mathrm{~K}$-gluconate, $5.0 \mathrm{EGTA}, 0.63 \mathrm{CaCl}_{2}$, $5.5 \mathrm{MgCl}_{2}, 10$ HEPES, $3 \mathrm{Na}_{2}$-ATP, $0.3 \mathrm{Na}_{3}-\mathrm{GTP}$, and 14 Tris-phosphocreatine ( $\mathrm{pH} 7.3,285-295$ mOsm). Voltage clamp recordings of spontaneous excitatory (sEPSCs) or inhibitory postsynaptic currents (sIPSCs) were made with internal solution containing (in mM) $133 \mathrm{CsCH}_{3} \mathrm{O}_{3} \mathrm{~S}, 3$ EGTA, $0.4 \mathrm{CaCl}_{2}, 5$ QX314, $4 \mathrm{MgCl}_{2}$, 10 HEPES, $3 \mathrm{Na}_{2}$-ATP, $0.3 \mathrm{Na}_{3}$-GTP (pH 7.3, 285$295 \mathrm{mOsm})$. Cells were voltage clamped at $-70 \mathrm{mV}$, the reversal potential of $\mathrm{Cl}^{-}$, to minimize the Cl-mediated sIPSCs thus to optimize the recording of sEPSCs. Cells were voltage clamped at $0 \mathrm{mV}$, the reversal potential of AMPA and NMDA receptormediated sEPSCs, to optimize recording of sIPSCs. Before bath application of the $\mathrm{GABA}_{\mathrm{A}}$ receptor blocker gabazine (GBZ), NBQX $(10 \mu \mathrm{M})$ and APV $(50 \mu \mathrm{M})$ were pre-applied to block fast glutamate receptors thus preventing epileptic activity.

\section{Electrical Stimulation}

Electrical stimulation was delivered by bipolar glass electrodes made from theta borosilicate tubes (Sutter Instrument). The isolated and constant current stimulation pulses (100 $\mu \mathrm{s})$ were triggered by a Master- 9 stimulator (AMPI, Jerusalem, Israel).

\section{Data Analysis}

Amplitudes and frequencies of sEPSCs or sIPSCs were measured with Wdetecta. ${ }^{1}$ Drug effects on sEPSCs and sIPSCs were determined by measuring these parameters from 1 min duration traces taken immediately before or $1 \mathrm{~min}$ (micropuffing) or $2 \mathrm{~min}$ (bath application) after drug application in each condition for each cell. Other data were measured and analyzed with Clampfit 11.0.3 (Molecular Devices) and Origin Pro 2019 (Origin Lab, Northampton, MA, United States). Statistical significance of population responses was calculated by using paired Student's $t$-test or ANOVA One-way repeated measure with Bonferroni post hoc comparisons in Origin Pro 2019.

\section{Drugs Delivery and Chemicals}

Drugs were applied by either bath perfusion to treat the whole slices or micropuffing to target the relevant glomeruli by $2 \mathrm{~ms}$ and 30 psi pneumatic pressure generated by a picospritzer (Parker Instruments, Cleveland, OH, United States). Micropuffing pipettes were made from thick wall borosilicate glass capillaries without filament (Sutter Instrument) with a 6 - $\mu \mathrm{m}$ tip diameter. These parameters were calibrated to deliver an injection volume of $\sim 20 \mathrm{nl}$ and were previously shown to deliver drug coverage restricted to a single glomerulus (Shao et al., 2012).

D-2-Amino-5-phosphonopentanoic acid sodium salt $\quad(A P V, \quad 50 \mu M), \quad 2,3-D i o x o-6-n i t r o-1,2,3,4-$ tetrahydrobenzo[f]quinoxaline-7-sulfonamide disodium salt (NBQX disodium salt, $10 \mu \mathrm{M}$ ), Gabazine (SR95531, $10 \quad \mu \mathrm{M}), \quad$ 8-Bromo-2,3,4,5-tetrahydro-3-methyl-5phenyl-1H-3-benzazepin-7-ol hydrobromide (SKF83566 bromide, $10 \mu \mathrm{M})$, (S)-(-)-5-Aminosulfonyl-N-[(1-ethyl-2pyrrolidinyl)methyl]-2-methoxybenzamide [(S)-(-)-Sulpiride, $100 \mu \mathrm{M}]$, (2S)-3-[[(1S)-1-(3,4-Dichlorophenyl)ethyl]amino-2hydroxypropyl](phenylmethyl)phosphinic acid hydrochloride (CGP55845 hydrochloride, $10 \mu \mathrm{M}$ ) were purchased from Tocris Cookson (Ellisville, MO, United States). Dopamine chloride (DA, $20 \mu \mathrm{M}$ or $100 \mu \mathrm{M}$ ) and all other chemicals were purchased from Sigma-Aldrich (St. Louis, MO, United States). All drugs were dissolved in distilled water as stock solution and diluted 1000 times with ACSF to final concentrations.

\section{RESULTS}

\section{Dopamine Inhibits Spontaneous EPSCs in Glomerular Interneurons via D1LRs}

Previous studies have shown that optogenetic activation of SACs leads to GABA and DA corelease (Liu et al., 2013). The coreleased GABA and DA orchestrate to modulate ETC physiological behaviors in such a way that DA enhances $I_{\mathrm{h}}$ to boost the rebound excitation following the $\mathrm{GABA}_{\mathrm{A}}$ receptormediated inhibition (Liu et al., 2013). Consistently, exogenous DA increases the $I_{\mathrm{h}}$-dependent bursts of action potentials in ETCs. As a major excitatory element in the glomerular circuit,

\footnotetext{
${ }^{1}$ https://hlab.stanford.edu/wdetecta.php
} 
ETCs monosynaptically drive vast majority of the inhibitory periglomerular cells (PGCs) and SACs (Shao et al., 2009; Kiyokage et al., 2010). All these findings lead us to predict that DA increases spontaneous excitatory synaptic activities in the ETC-driven PGCs and SACs. To test this, we recorded sEPSCs in PGCs and SACs in OB slices under voltage clamp and examined effects of exogenous DA. As PGCs and SACs exclusively express the key enzyme of GABA biosynthesis GAD65 and the ratelimiting enzyme of dopamine biosynthesis tyrosine hydroxylase (TH), respectively (Kiyokage et al., 2010), PGCs and SACs were accordingly identified in GAD65-GFP (Figure 1A) and TH-GFP (not shown) mice. To differentiate the ON-driven and ETCdriven PGCs and SACs, we started with recording their responses to $\mathrm{ON}$ stimulation. Consistent with previous findings (Shao et al., 2009; Kiyokage et al., 2010), vast majority of PGCs (8/13) or SACs $(8 / 12)$ respond to $\mathrm{ON}$-stimulation with long and inconsistent latencies ( $4.3 \pm 0.31 \mathrm{~ms}, n=8$ PGCs; $4.6 \pm 0.42, n=8$ SACs) EPSCs and exhibit bursts of sEPSCs (Figures 1B,C), indicating that they are ETC-driven glomerular interneurons. The rest PGCs (5/13) and SACs (4/12) responded to ON stimulation with short latencies $(2.3 \pm 0.15 \mathrm{~ms}, n=5$ PGCs; $2.1 \pm 0.17$, $n=4$ SACs), suggesting that they are ON-driven glomerular interneurons. Surprisingly, bath application of DA reversibly suppressed sEPSCs in all tested PGCs $(n=13$, Figures 1C$\mathbf{F}, \mathbf{H})$ and SACs $(n=12)$. sEPSC frequency and amplitude in PGCs $(n=13)$ were $63.0 \pm 6.7 \mathrm{~Hz}$ and $57.4 \pm 5.0 \mathrm{pA}$ in ACSF, $29.2 \pm 3.0 \mathrm{~Hz}(P<0.0001$ vs. in ACSF $)$ and $32.3 \pm 3.4 \mathrm{pA}$ $(P<0.0001$ vs. in ACSF $)$ in DA $(20 \mu \mathrm{M})$, and $61.3 \pm 6.3 \mathrm{~Hz}$ $(P=1$ vs. in ACSF $)$ and $55.7 \pm 5.0 \mathrm{pA}(P=1$ vs. in ACSF $)$ after DA washout. Similarly, sEPSC frequency and amplitude in 12 SACs were $64.3 \pm 8.4 \mathrm{~Hz}$ and $71.0 \pm 9.0 \mathrm{pA}$ in ACSF, $28.8 \pm 3.6$ $(P<0.0001$ vs. ACSF $)$ and $37.4 \pm 4.3 \mathrm{pA} \mathrm{Hz}(P<0.0001$ vs. $\mathrm{ACSF})$ in DA, $63.6 \pm 8.5 \mathrm{~Hz}(P=1 \mathrm{vs}$. ACSF $)$ and $70.3 \pm 8.8 \mathrm{pA}$ $(P=1$ vs. ACSF) after DA washout (Figures $\mathbf{1 I}, \mathbf{K})$. While the DA suppression of sEPSCs in the ON-driven PGCs and SACs is consistent with previous findings that DA inhibits glutamate release from the ON terminals (Hsia et al., 1999; Berkowicz and Trombley, 2000; Ennis et al., 2001; Maher and Westbrook, 2008; Vaaga et al., 2017), the similar DA action on the ETCdriven PGCs and SACs may indicate dopaminergic modulation of glutamate release from ETC apical dendritic terminals in the glomerular layer. Given that burst sEPSCs reflect spontaneous burst firing activities in presynaptic ETCs, we also measured the sEPSC burst frequencies and found that the frequency of sEPSC bursts was also reduced by DA (Figures 1G,J). sEPSC burst frequencies in PGCs $(n=8)$ and SACs $(n=8)$ were $6.1 \pm 1.0 \mathrm{~Hz}$ and $7.8 \pm 1.1 \mathrm{~Hz}$ in ACSF, $4.1 \pm 0.7 \mathrm{~Hz}(P<0.0001$ vs. ACSF $)$ and $5.3 \pm 0.8 \mathrm{~Hz}(P<0.0001$ vs. ACSF $)$ in DA, $6.1 \pm 0.9 \mathrm{~Hz}$ $(P=1$ vs. $\mathrm{ACSF})$ and $7.7 \pm 1.0 \mathrm{~Hz}(P=1$ vs. ACSF $)$ after DA washout, respectively. Collectively, these results indicate that DA inhibits glutamate release from presynaptic ETC apical dendrites to PGCs and SACs thus reduces burst sEPSCs.

Dopamine actions are mediated by two major categories of G protein-coupled receptors - D1LRs and D2LRs (Beaulieu and Gainetdinov, 2011). Evidence shows that D2LRs in ON terminals mediate presynaptic inhibition of glutamate release to their postsynaptic targets in the glomerular layer (Nickell et al., 1991;
Aroniadou-Anderjaska et al., 2000; McGann, 2013). This explains why DA suppresses sEPSCs in the ON-driven PGCs and SACs. All ETCs receive direct ON input (Hayar et al., 2004a; De Saint et al., 2009; Gire and Schoppa, 2009), does DA inhibit ON terminals via D2LRs to reduce ETC burst firing thus indirectly suppresses burst sEPSCs in the ETC-driven PGCs and SACs? To test this, we treated OB slices with bath application of (S)-()-Sulpiride $(100 \mu \mathrm{M})$, a selective D2LR antagonist, for $10 \mathrm{~min}$ before addition of DA (Figure 2A). In these conditions, DA reduced sEPSC frequency by $46.8 \pm 1.9 \%(n=7)$ in PGCs and $49.7 \pm 1.9 \%(n=6)$ in SACs, amplitude by $47.9 \pm 3.7 \%(n=7)$ in PGCs and $48.3 \pm 3.0 \%(n=6)$ in SACs (Figures 2A-C) in the presence of (S)-(-)-Sulpiride. Specifically, sEPSC frequency and amplitude in PGCs $(n=7)$ were $76.8 \pm 11.9 \mathrm{~Hz}$ and $75.1 \pm 11.6$ pA in (S)-(-)-Sulpiride, $40.3 \pm 5.9 \mathrm{~Hz}[P<0.0001$ vs. (S)-(-)Sulpiride $]$ and $39.0 \pm 6.8 \mathrm{pA}[P<0.0001$ vs. (S)-(-)-Sulpiride $]$ in (S)-(-)-Sulpiride+DA, and $77.4 \pm 12.4 \mathrm{~Hz}[P=1$ vs. (S)-(-)Sulpiride $]$ and $74.0 \pm 11.7$ pA [ $P=1$ vs. (S)-(-)-Sulpiride] after washout DA, respectively (Figures 2D,E). Similar results were observed in SACs $(n=6)$ with average frequency and amplitude of $68.2 \pm 11.4 \mathrm{~Hz}$ and $65.7 \pm 12.1 \mathrm{pA}$ in (S)-(-)-Sulpiride, $34.2 \pm 6.0 \mathrm{~Hz}[P<0.0001$ vs. (S)-(-)-Sulpiride $]$ and $34.3 \pm 6.7 \mathrm{pA}$ $[P<0.0001$ vs. (S)-(-)-Sulpiride] in the addition of DA, and $67.1 \pm 12.0 \mathrm{~Hz}[P=1$ vs. (S)-(-)-Sulpiride] and $64.3 \pm 11.8 \mathrm{pA}$ $[P=1$ vs. (S)-(-)-Sulpiride] after DA washout (Figures 2D,E). These findings suggest that D2LRs do not mediate presynaptic inhibition of glutamate release from ON terminals to ETCs to PGCs and SACs.

Then we examined DA effects on sEPSCs in PGCs and SACs in the presence of the selective D1LR antagonist SKF83566 (10 $\mu \mathrm{M})$. However, in these conditions, DA altered neither frequency nor amplitude of sEPSCs in either population of glomerular interneurons (Figures 2F-J), implying that the DA suppression of sEPSCs in both PGCs and SACs is mediated by D1LRs.

Altogether, our results support that DA reduces sEPSCs in the ETC-driven PGCs and SACs by activation of D1LRs but this action is not due to reduced ETC activities, which result from activation of D2LRs on ON terminals to reduce glutamate release.

\section{DA Enhances Spontaneous IPSCs in Glomerular Neurons}

Since activation of D1LRs on ETCs produces an excitatory effect (Liu et al., 2013) whereas ON terminals are devoid of D1LRs (Levey et al., 1993; Coronas et al., 1997; Beaulieu and Gainetdinov, 2011), we hypothesized that the DA via D1LRs directly excites PGCs and/or SACs to release GABA, which activates $\mathrm{GABA}_{\mathrm{B}}$ receptors on ETC apical dendrites (Karpuk and Hayar, 2008) to reduce glutamate release onto PGCs and SACs. In this scenario, DA should elevate the glomerular inhibition. To test this, we recorded spontaneous inhibitory postsynaptic currents (sIPSCs) in voltage clamp in all three populations of glomerular neurons including PGCs, SACs and ETCs, which all have apical dendrites or processes confined to the glomerular layers thus could manifest potential DA-enhanced glomerular inhibition. To maximize detection sIPSCs, which have reversal potential around $-70 \mathrm{mV}$ based on chloride concentrations of our internal and 
A

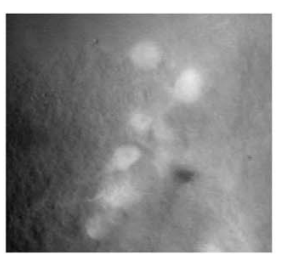

C

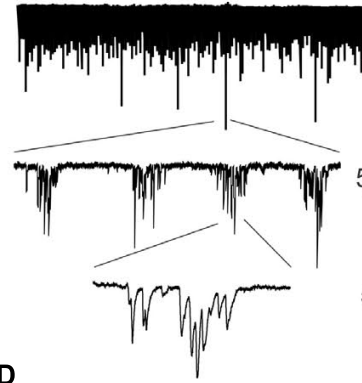

D

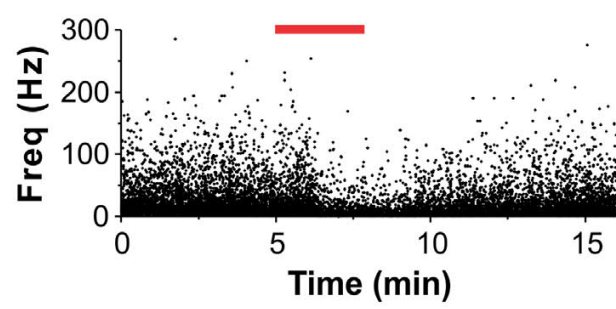

F

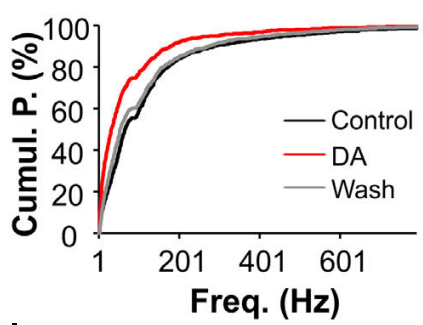

I

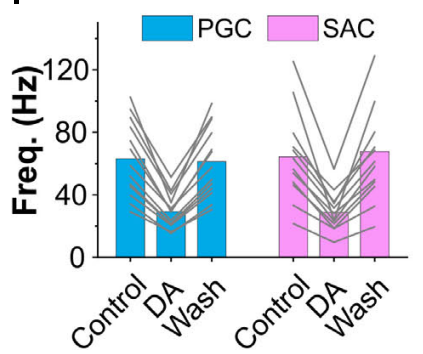

B

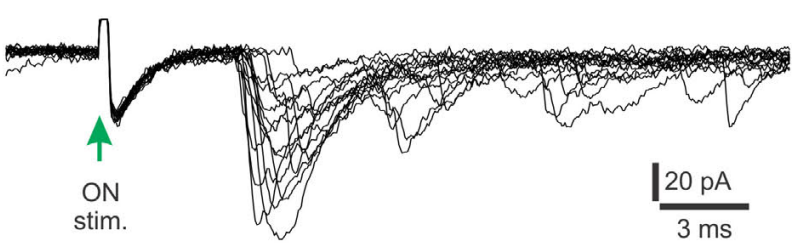

DA
G

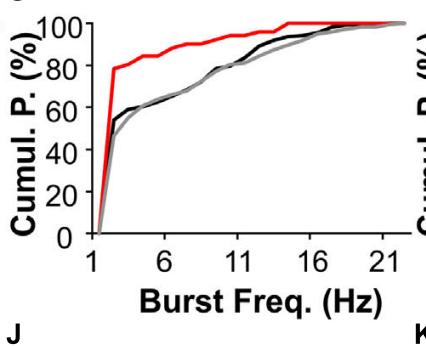

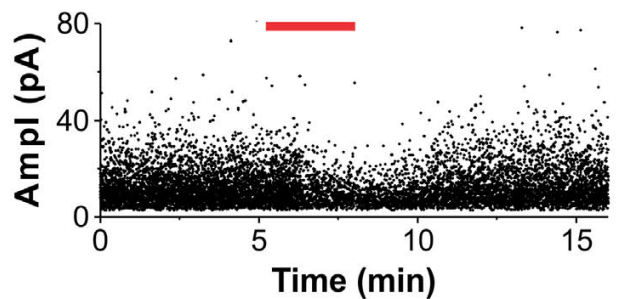

H

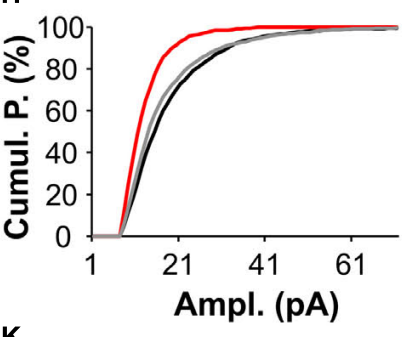

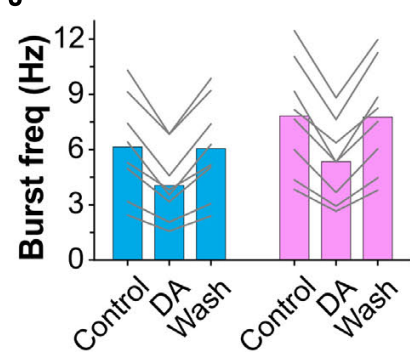

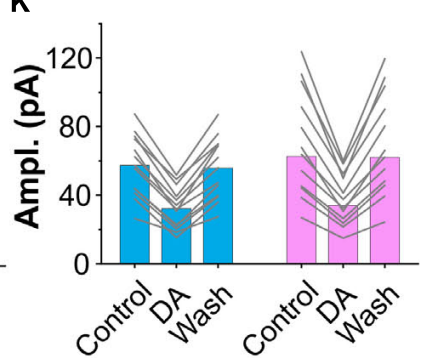

FIGURE 1 | DA suppresses sEPSCs in glomerular interneurons. (A) Photo graph showing GFP-expressing periglomerular cells (PGCs) in the glomerular layer of an OB slice prepared from a GAD65-GFP mouse. (B) Typical excitatory postsynaptic current (EPSCs) traces in a PGC evoked by stimulation of the olfactory nerves (ON-stim.). Note that EPSC onset latencies are longer than $3 \mathrm{~ms}$ and varies among traces. (C) A representative voltage clamp recording trace showing that bath-applied DA (20 $\mu \mathrm{M}$, red horizontal bar) reduces both frequency and amplitude of spontaneous burst EPSCs in a PGC (top). Middle and bottom traces are blown-up. (D,E): plotting graphs showing changes in instant frequency (D) and amplitude (E) of sEPSCs before, during and after DA application in the same trace in (C). (F-H) Line graphs of cumulative probability (Cumul. P.) against frequency of individual (F) or burst (G) sEPSCs, or amplitude (Ampl., H) of individual sEPSCs before (control), during (DA), and after (wash) DA application. (I-K) Pooled data showing that DA reduces both frequency of individual (I) or burst (J) sEPSCs and amplitude (K) of sEPSCs in both PGCs $(n=13)$ and short-axon cells (SACs, $n=12)$.

external solutions, and minimize sEPSCs, which have a reversal potential at $0 \mathrm{mV}$, we voltage clamped cells at $0 \mathrm{mV}$ in the presence of NBQX $(10 \mu \mathrm{M})$ and D-APV $(50 \mu \mathrm{M})$ to block AMPA and NMDA receptors thus minimize circuit influence. In these conditions, bath application of DA $(20 \mu \mathrm{M})$ reversibly enhanced sIPSCs in PGCs (Figure 3A). When sIPSCs were completely 

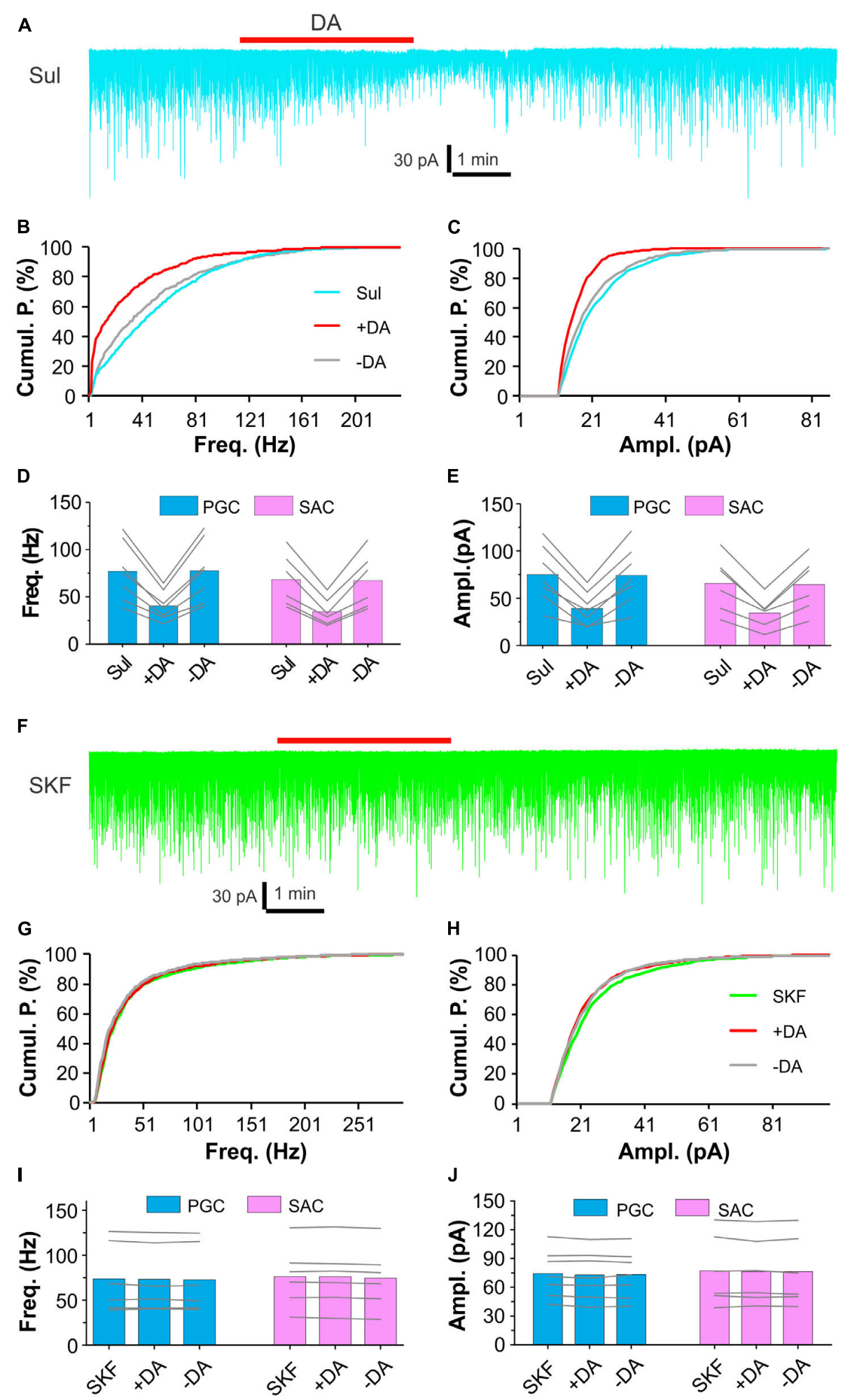

FIGURE 2 | D1LRs mediate DA actions on sEPSCs in glomerular interneurons. (A) A representative recording trace showing DA effects on sEPSCs in a PGC in the presence of $100 \mu \mathrm{M}(\mathrm{S})-(-)-$ Sulpiride (Sul), a selective D2LR antagonist. (B,C) Graphs plotting cumulative probability against frequency (B) or amplitude (C) of sEPSCs shown in (A). (D,E) Pooled data showing that DA reduces both frequency (D) and amplitude (E) of sEPSCs in both PGCs $(n=7)$ and SACs $(n=6)$ in the presence of (S)-(-)-Sulpiride. (F) A representative recording trace showing that DA has no effect on sEPSCs in a PGC in the presence of $10 \mu \mathrm{M}$ SKF83566 (SKF), a selective D1LR antagonist. (G,H) Graphs plotting cumulative probability against frequency $\mathbf{( G )}$ or amplitude $\mathbf{( H )}$ of sEPSCs shown in $\mathbf{( F )}$. (I,J) Pooled data showing that DA has effect on neither frequency (I) nor amplitude (J) of sEPSCs in either PGCs $(n=7)$ or SACs $(n=6)$ in the presence of SKF83566. 


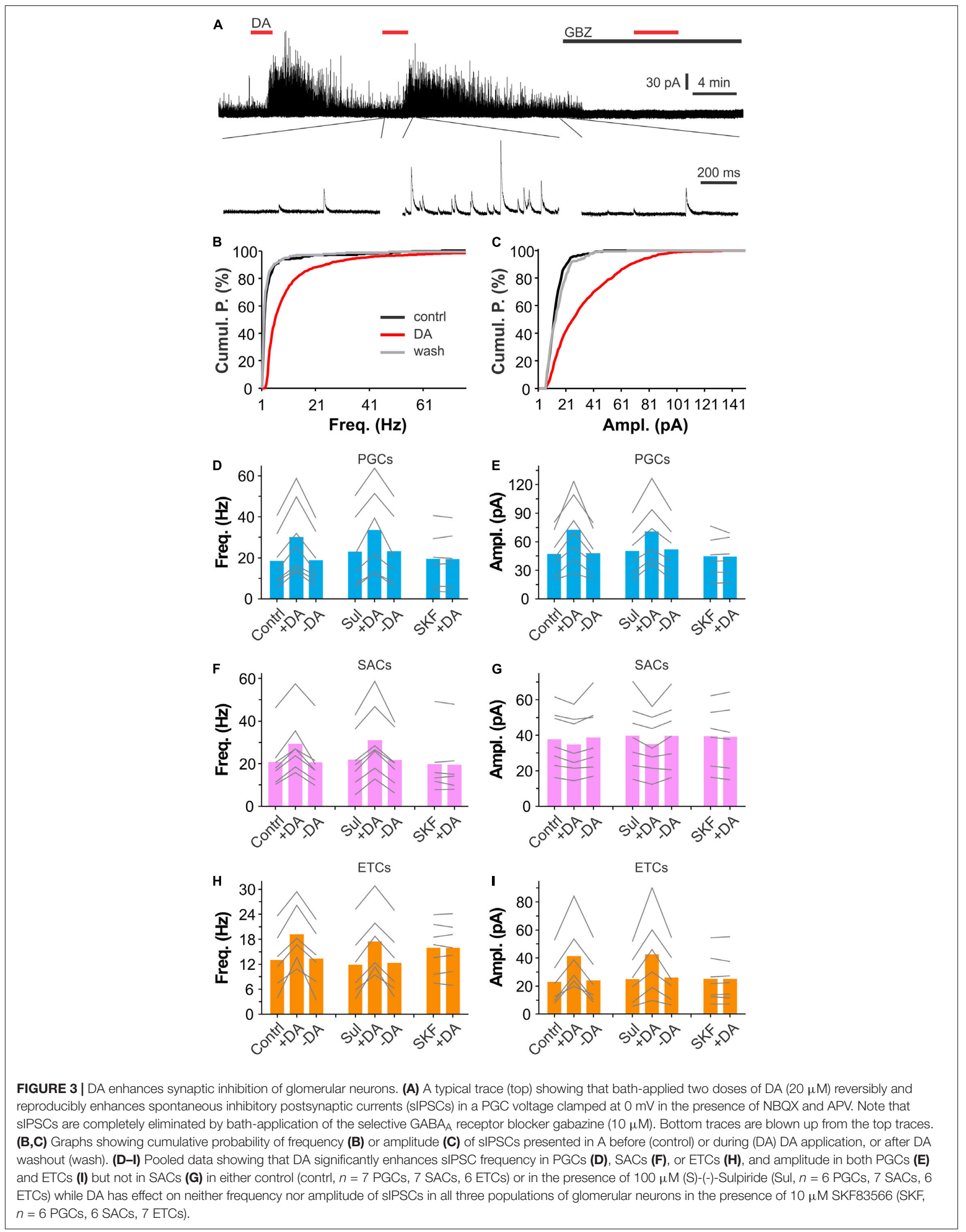


abolished by the selective $\mathrm{GABA}_{\mathrm{A}}$ receptor blocker gabazine (GBZ, $10 \mu \mathrm{M}$, Figure 3A), DA showed no effect on the holding current, suggesting that DA does not affect PGCs per se. Further analysis revealed that both frequency and amplitude of sIPSCs in PGCs were elevated by DA (Figures 3B,C). To determine what receptors mediate these actions, either (S)-(-)-Sulpiride $(100 \mu \mathrm{M})$ or SKF83566 $(10 \mu \mathrm{M})$ was bath-applied before DA addition in another set of experiments. It turned out that SKF83566 rather than (S)-(-)-Sulpiride completely blocked DA effects on sIPSCs in all tested PGCs (Figures 3D,E), indicating mediation by D1LRs. Specifically, the average frequency and amplitude of sIPSCs in seven PGCs were $18.4 \pm 5.0 \mathrm{~Hz}$ and $47.1 \pm 8.7 \mathrm{pA}$ in ACSF, $30.0 \pm 6.8 \mathrm{~Hz}(p<0.0001$ vs. in ACSF $)$ and $72.5 \pm 13.4 \mathrm{pA}$ in DA $(p<0.0001$ vs. in ACSF), and $18.8 \pm 4.8 \mathrm{~Hz}(p=1$ vs. in ACSF) and $47.9 \pm 8.5 \mathrm{pA}$ ( $p=1$ vs. in ACSF) after DA washout, respectively. In the presence of (S)-(-)-Sulpiride, average frequency and amplitude of sIPSCs in another set of six PGCs were $23.0 \pm 7.5 \mathrm{~Hz}$ and $50.2 \pm 11.0 \mathrm{pA}, 33.6 \pm 8.7 \mathrm{~Hz}$ $(P<0.0001$ vs. Sul $)$ and $70.7 \pm 14.1 \mathrm{pA}(P<0.0001$ vs. Sul $)$ with addition of DA, $23.2 \pm 7.3 \mathrm{~Hz}(p=1 \mathrm{vs}$. Sul) and $51.8 \pm 11.1 \mathrm{pA}$ ( $p=1$ vs. Sul), respectively. However, with SKF83566 in the bath, average frequency and amplitude of sIPSCs in six PGCs were $19.5 \pm 5.7 \mathrm{~Hz}$ and $44.6 \pm 9.0 \mathrm{pA}, 19.4 \pm 5.6 \mathrm{~Hz}(p=1$ vs. $\mathrm{SKF}$ ) and $44.4 \pm 8.3 \mathrm{pA}$ ( $p=1$ vs. SKF) with addition of DA, respectively. Similar pharmacological results of DA actions on
sIPSCs were observed in both SACs and ETCs (Figures 3F-I) except DA did not show significant effect on sIPSC amplitude in SACs (Figure 3G), supporting our prediction that DA increases overall glomerular inhibition.

In sum, our results demonstrate that DA enhances both frequency and amplitude of sIPSCs similarly in both PGCs and ETCs while in SACs DA increased only frequency but not amplitude of sIPSCs. Pharmacological analyses suggest that all these elevating effects in all three populations of glomerular neurons are mediated by D1LRs.

\section{Glomerular Application of DA Augments sIPSCs in Mitral Cells (MCs)}

MCs, the principal OB output neurons, have apical dendrites ramifying in single glomeruli where they receive glomerular inhibition. Thus, it was plausible to predict that DA potentiated glomerular inhibition of MCs. To examine this possibility, we voltage clamped MCs at $-0 \mathrm{mV}$ in OB slices to maximize sIPSC detection in the presence of NBQX and APV. MC responses to DA $(100 \mu \mathrm{M})$ micropuffed (30 psi for $2 \mathrm{~ms}$ ) to the glomeruli receiving apical dendrites of the recorded cells were recorded as described previously (Shao et al., 2012). Alexa $594(10 \mu \mathrm{M})$ was included in internal solution to visualize the recorded cells and their apical dendrites thus facilitate DA micropuffing

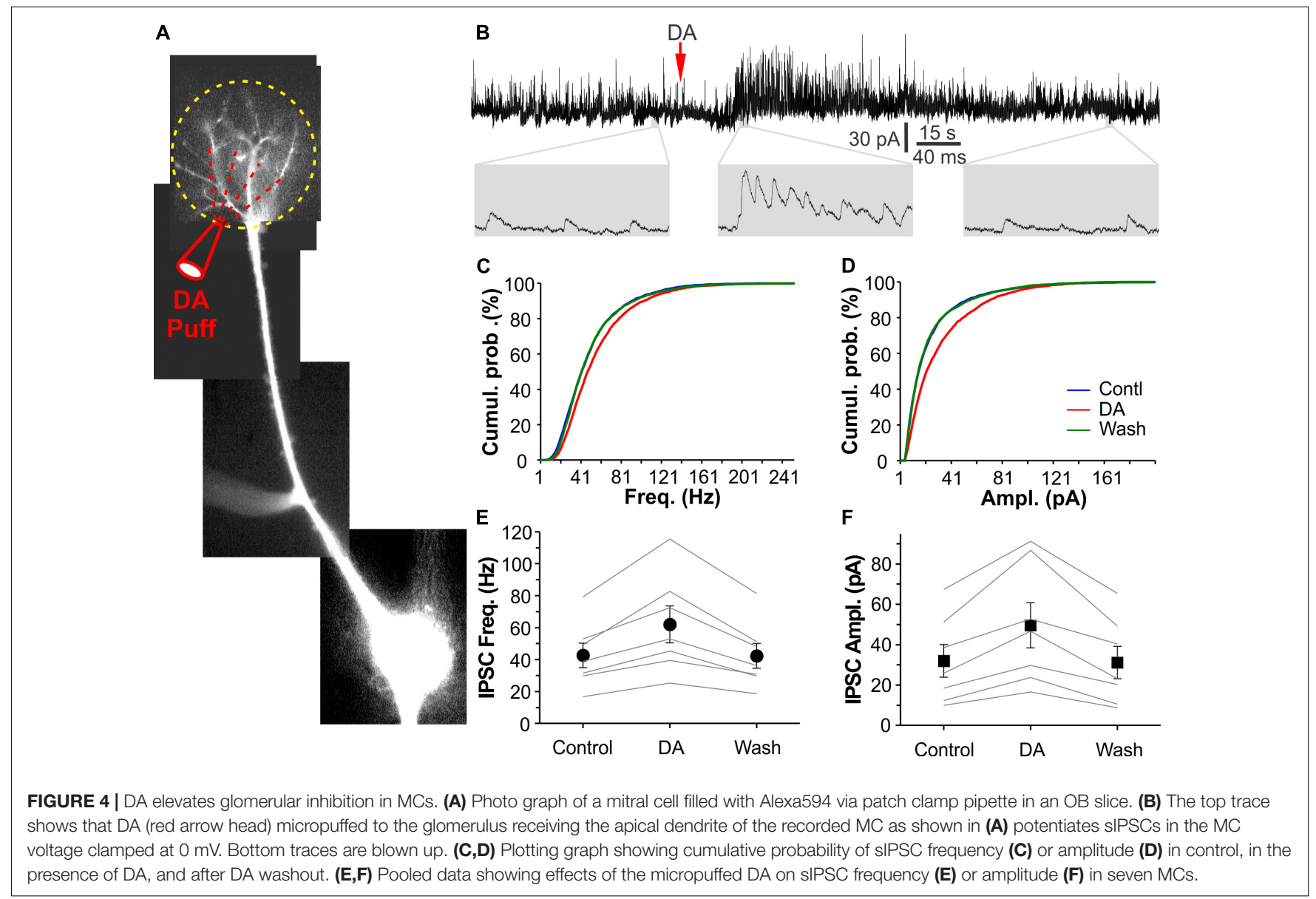


(Figure 4A). In these conditions, micropuffing DA reversibly increased both frequency and amplitude of sIPSCs in all tested seven MCs (Figures 4B-F). The average frequency and amplitude of sIPSCs in seven cells were $42.6 \pm 7.7 \mathrm{~Hz}$ and $32.0 \pm 8.7 \mathrm{pA}$ in ACSF (control), $62.0 \pm 11.6 \mathrm{~Hz}$ ( $p<0.001$ vs. control) and $49.6 \pm 11.2 \mathrm{pA}(p<0.001$ vs. control $)$ in $\mathrm{DA}$, and $42.3 \pm 7.8 \mathrm{~Hz}$ ( $p=1$ vs. control) and $31.1 \pm 8.0 \mathrm{pA}$ ( $p=1$ vs. control) after DA washout, respectively. Collectively, our results demonstrate that DA enhances glomerular inhibition of MCs.

\section{Dopamine Selectively Excites Short Axon Cells}

Periglomerular cells and SACs are two major populations of inhibitory glomerular interneurons. To test whether DA activates them to release GABA and increase glomerular inhibition, we did both voltage and current clamp recordings from both SACs and PGCs in the presence of synaptic transmission blockers NBQX $(10 \mu \mathrm{M}), \operatorname{APV}(50 \mu \mathrm{M})$ and GBZ $(10 \mu \mathrm{M})$ to eliminate circuit influence. SACs responded to bath application of DA with an inward current (Figure 5A) in voltage clamp $\left(V_{\text {hold }}=-65 \mathrm{mV}\right)$ or depolarization with spikes in current clamp (Figure 5C) while PGCs did not respond to the same dose of DA (Figures 5B,D). The average amplitude of the DA-induced inward current in five SACs was $13.0 \pm 2.6 \mathrm{pA}$ in ACSF and $0.2 \pm 0.1 \mathrm{pA}$ in the presence of SKF83566 ( $p<0.01$ vs. in ACSF) while in five PGCs the average amplitude was $0.4 \pm 0.1 \mathrm{pA}$ (Figure 5E). In current clamp, the average amplitude of DA-induced depolarization in five SACs was $5.1 \pm 0.6 \mathrm{mV}$ in ACSF and $0.3 \pm 0.1 \mathrm{mV}(p<0.01$ vs. in ACSF $)$ in the presence of SKF83566 while there was no depolarization in five PGCs $(0.2 \pm 0.1 \mathrm{mV})$ (Figure 5F). Taken together, our results demonstrate that DA selectively excites SACs via D1LRs.

\section{GABA $_{B}$ Receptors Mediate the DA Suppressive Effects on sEPSCs in Glomerular Interneurons}

Dopamine and GABA are coreleased from SACs (Maher and Westbrook, 2008; Borisovska et al., 2013; Liu et al., 2013;

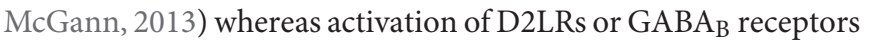
produces presynaptic inhibition in many brain areas including the OB (Nickell et al., 1994; Aroniadou-Anderjaska et al., 2000; Ennis et al., 2001; Isaacson and Vitten, 2003; Wachowiak et al., 2005; Beaulieu and Gainetdinov, 2011; McGann, 2013). To test whether activation of $\mathrm{GABA}_{\mathrm{B}}$ receptors is required for the DA actions we observed, sEPSCs were recorded in both PGCs and SACs and their responses to DA were compared between with and without the selective $\mathrm{GABA}_{\mathrm{B}}$ receptor antagonist CGP55845 $(10 \mu \mathrm{M})$ in the bath. As shown in Figures 6A-C, neither CGP55845 nor CGP55845 plus DA showed effect on sEPSCs in a PGC compared to control. Further analysis revealed DA effect on neither frequency nor amplitude of sEPSCs in six PGCs or

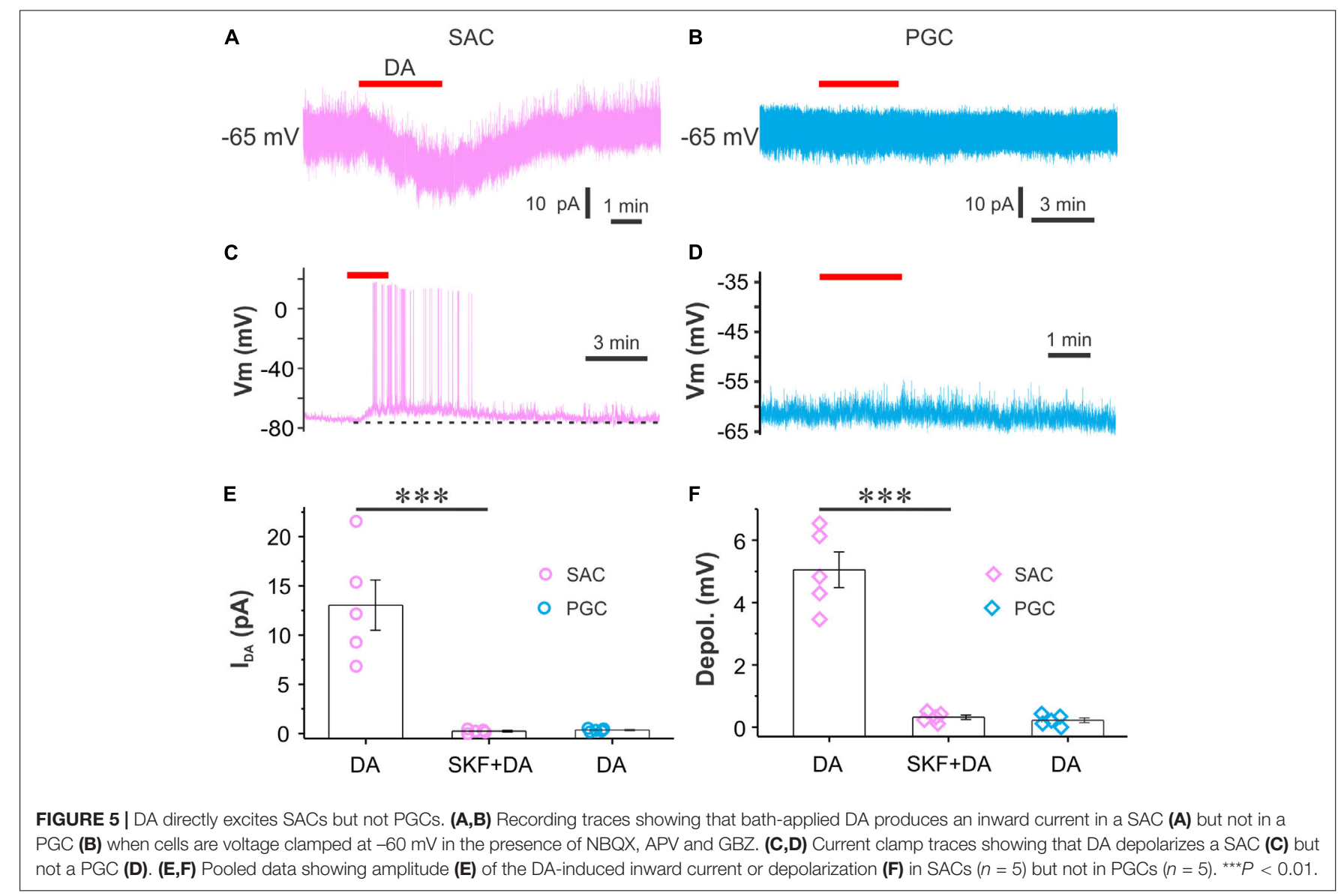



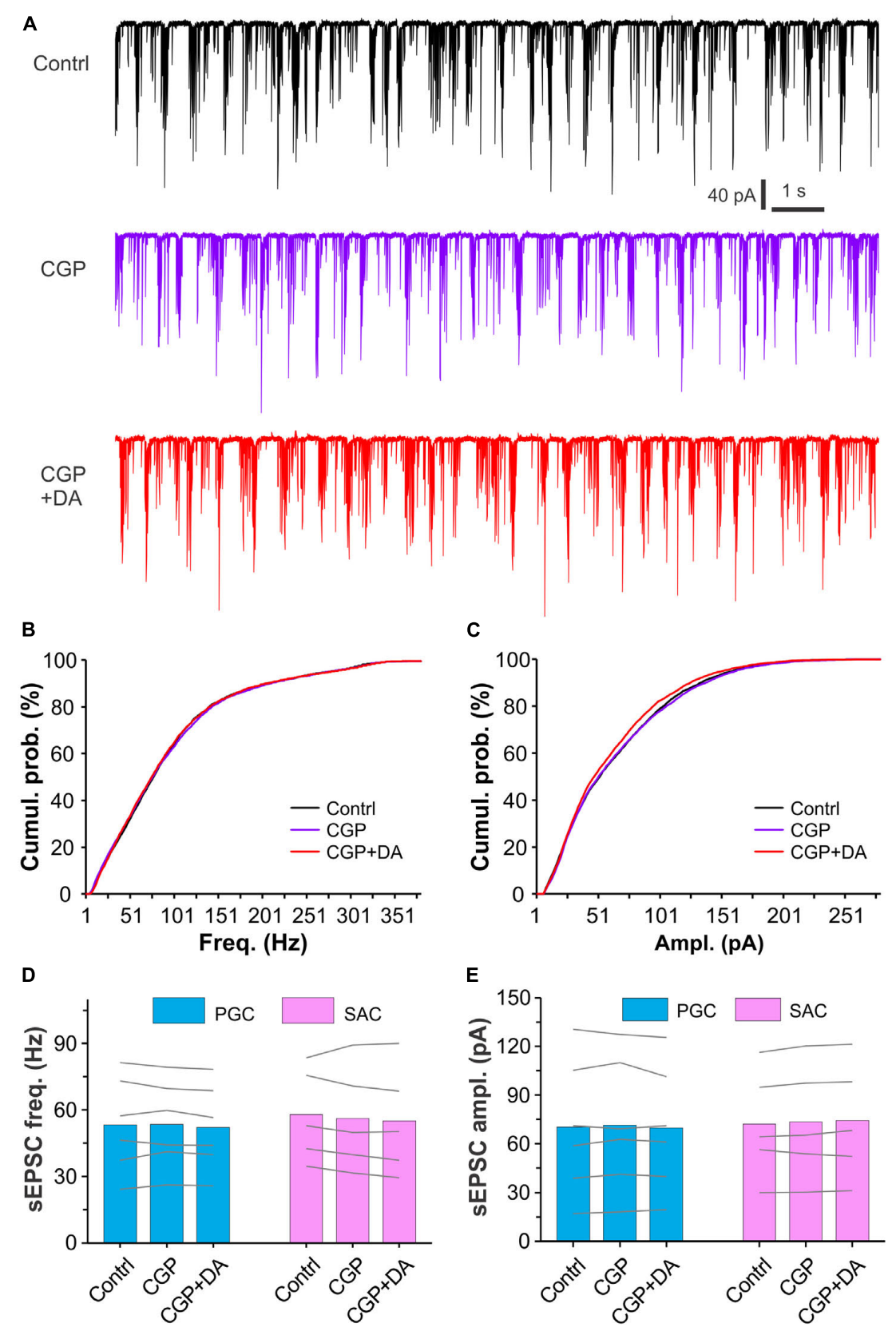

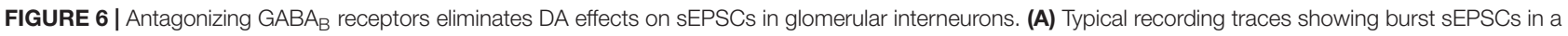
PGC before (top), during (middle) bath application of CGP55845 (CGP, $10 \mu \mathrm{M}$ ), a selective GABA $A_{B}$ receptor antagonist, or addition of DA (bottom) in the presence of CGP. (B,C) Graphs showing cumulative probability of frequency (B) or amplitude (C) of sEPSCs presented in (A). (D,E) Pooled data showing that DA has effect on neither frequency (D) nor amplitude (E) of sEPSCs in either PGCs $(n=6)$ or SACs $(n=5)$ in the presence of CGP55845.

five SACs (Figures 6D,E), suggesting that activation of GABA receptors is required for the DA suppressive effects on sEPSCs in PGCs and SACs.

\section{DA Produces an Excitatory Net Effect on MCs}

External tufted cells provide direct excitatory input to MCs and contribute to long-lasting depolarization (LLD) (De Saint et al., 2009; Gire and Schoppa, 2009). Our previous study showed that DA increases ETC spontaneous spike burst frequency (Liu et al., 2013), indicating that DA increase LLD frequency. However, our present results showed that DA enhanced glomerular inhibition at least partially due to its selective activation of SACs, indicating that DA inhibits MC output. What will be the DA net effects on MC output with integration of these two directionally opposite actions? To answer this question, we first recorded MCs in voltage clamp with a holding potential at $-60 \mathrm{mV}$ and observed their responses 

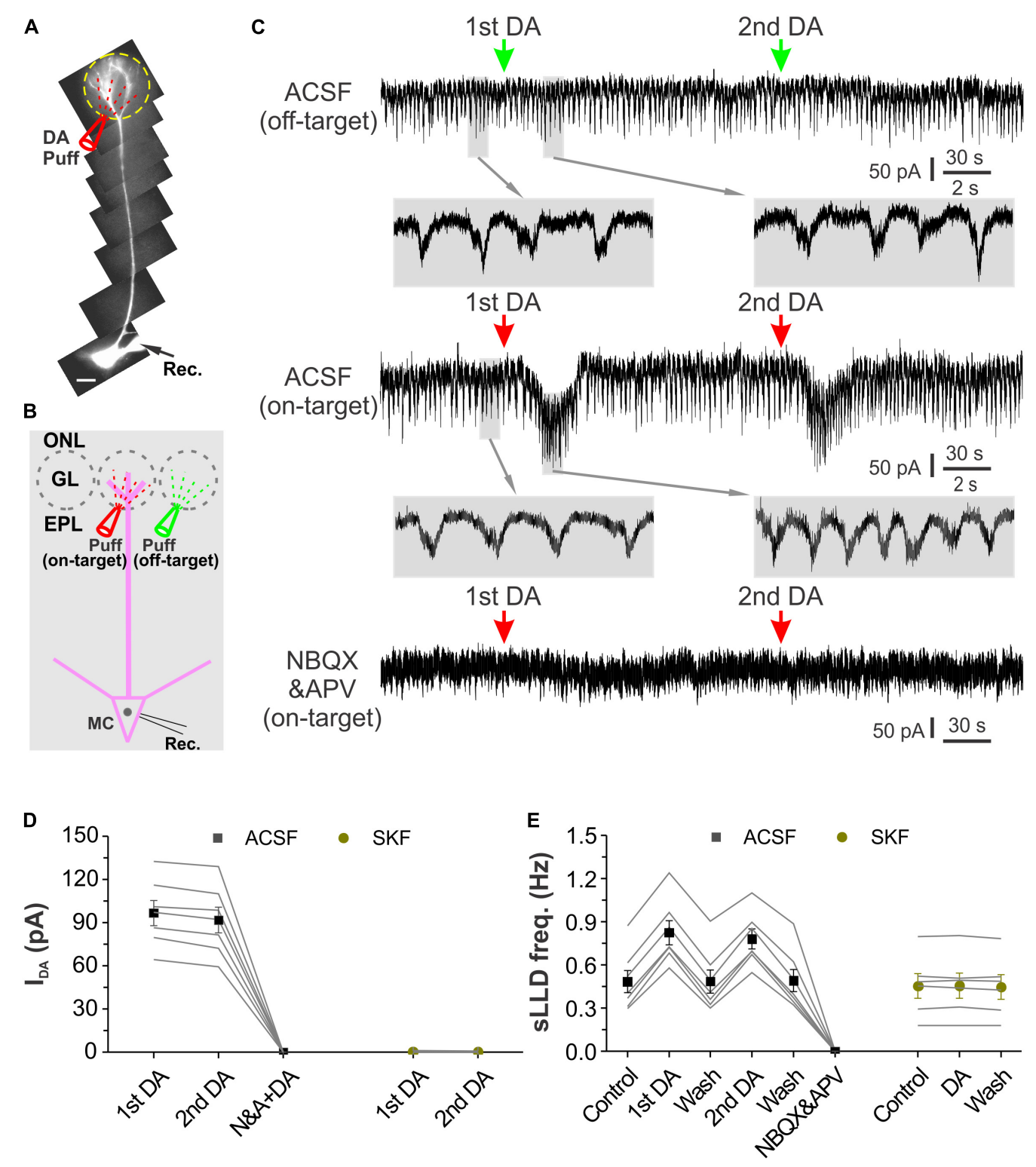

FIGURE 7 | Glomerular application of DA produces an inward current and increases SLLD frequency in MCs. (A) Photo graph of a MC filled with Alexa594 via patch clamp pipette in an OB slice. (B) Diagram illustration of DA application to the glomerulus (on-target) receiving the apical dendrites of the recorded MC or its adjacent glomerulus (off-target). (C) Recording traces showing the rhythmic long-lasting depolarizing (LLD)/inward currents recorded in a MC voltage clamped at -60 mV and their responses to off-target (top), on-target application of DA as shown in (B) in control condition (middle) or in the presence of NBQX and APV to block fast glutamate receptors (bottom). Traces at expanded time scale beneath the top and middle traces show changes in LLD current frequency after DA application. (D) Graphs of pooled data from two groups of MCs. Data of the first group of MCs $(n=7)$ show the amplitude of the inward currents induced by the first (1st) and second (2nd) dose of DA in ACSF, in the presence of NBQX and APV (N\&A). The second group of MCs $(n=6)$ show effects of two doses of DA on-target application on the holding current in the presence of SKF83566, a selective D1LR antagonist. (E) Pooled data from two groups of MCs. Data of the first group of MCs ( $n=7$ ) show the frequency of the spontaneous LLD (sLLD) currents in ACSF (control), addition of the first dose of dopamine (1st DA), washout of the first dose of DA, application of the second dose of DA (2nd DA), washout of the second dose of DA, or in the presence of NBQX and APV. Data of the second group of MCs $(n=6)$ show sLLD frequency in the presence of SKF83566 before (control) or during (DA) DA application, and after DA washout (wash).

to glomerular application of DA as shown by Figures 7A,B. Alexa $594(10 \mu \mathrm{M})$ was included in the patch pipette to visualize the recorded $\mathrm{MC}$ somata and their apical dendrites. In these conditions, micropuffing DA (100 $\mu \mathrm{M}, 2 \mathrm{~ms})$ to glomeruli (off-target) next to the ones receiving the apical dendrites of the recorded cells did not evoke alteration in the spontaneous
LLD (sLLD, Figure 7C top traces). However, when the puffing electrode was moved to target the glomerulus innervated by the apical dendrite of the recorded cell (on-target), DA reversibly induced an inward current in MCs (Figure 7C middle traces). The frequency of the spontaneous LLD superimposed on the inward current was increased compared to that before DA 


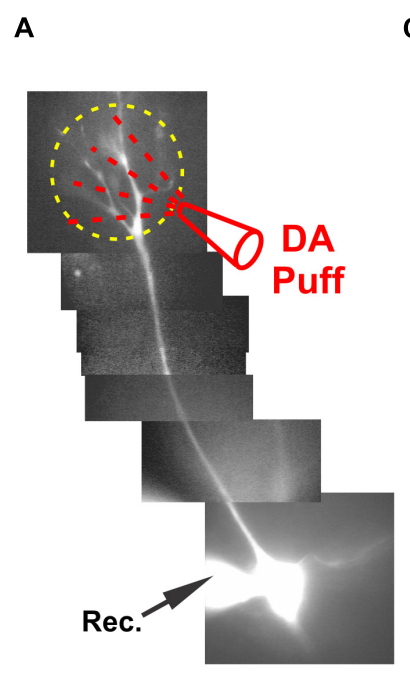

B
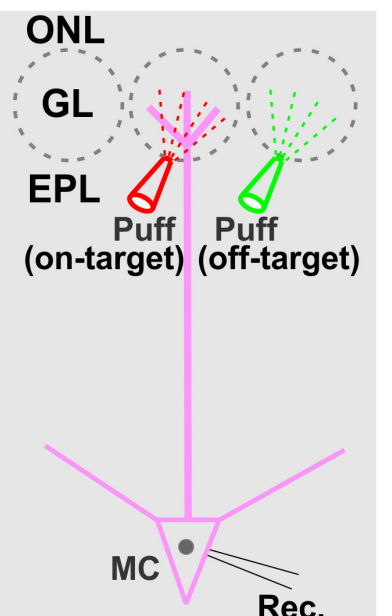

C

ACSF

(off-target)
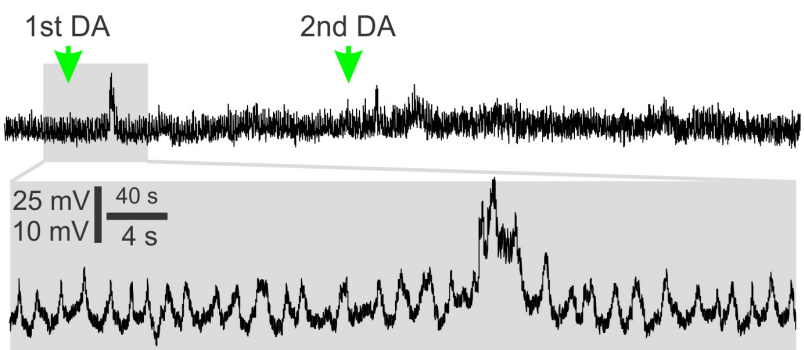

1st DA 2nd DA

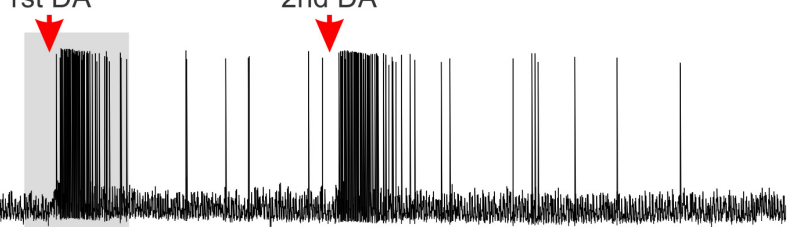

$30 \mathrm{mV} \frac{40 \mathrm{~s}}{4 \mathrm{~s}}$

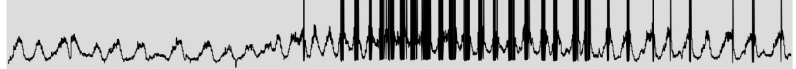

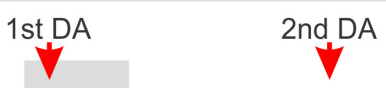

SKF

(on-target)

$25 \mathrm{mV} 40 \mathrm{~s}$

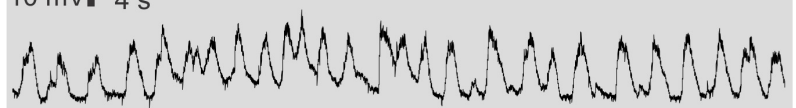

1st DA 2nd DA

NBQX\&APV

(on-target)
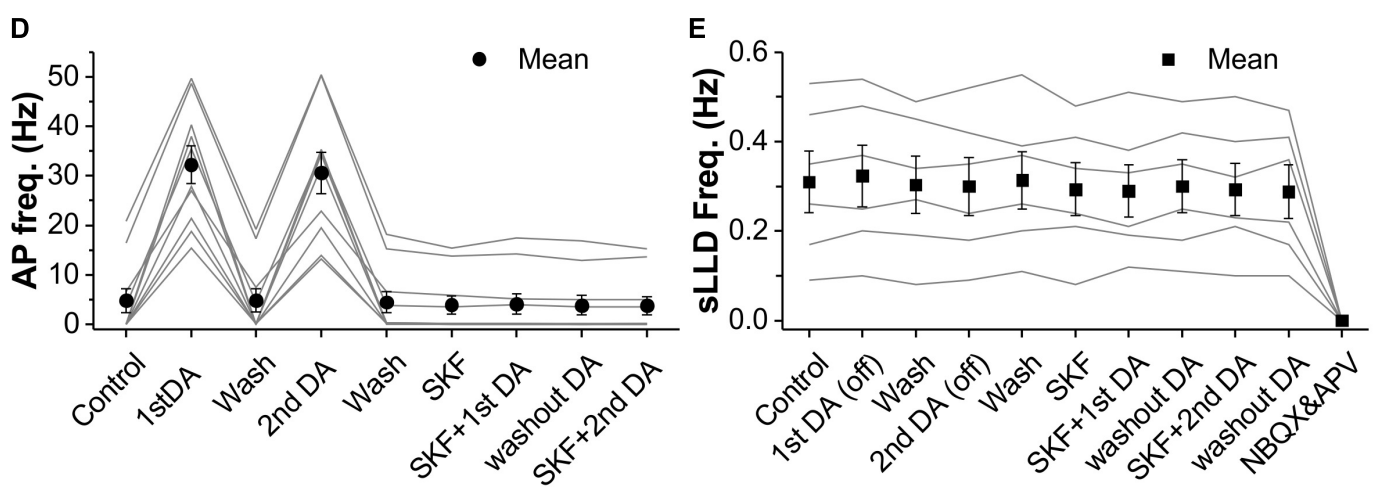

FIGURE 8 | Glomerular application of DA elevates OB output from MCs. (A) Photo graph of a MC filled with Alexa594 via patch clamp pipette in an OB slice. (B) Diagram illustration of DA application to the glomerulus (on-target) receiving the apical dendrites of the recorded MC or its adjacent glomerulus (off-target). (C) Recording traces showing the spontaneous rhythmic long-lasting depolarization (LLD) recorded in a MC in current clamp and their responses to off-target (top) or on-target application of DA as shown in (B) in ACSF, in the presence of SKF83566 to block D1LRs (middle) or NBQX and APV to block fast glutamate receptors (bottom). sLLDs are completely eliminated by NBQX and APV. Blown-up traces show DA effects on sLLD frequency or spike firing in each condition. (D) Graphs of pooled data from $10 \mathrm{MCs}$ showing effects of two doses of on-target DA on action potential (AP) frequency in ACSF or in the presence of SKF83566. (E) Graphs of pooled data from six MCs showing effects of two doses of off-target DA or two doses of on-target DA on sLLD frequency in the presence of SKF83566 as well as effect of NBQX and APV. 


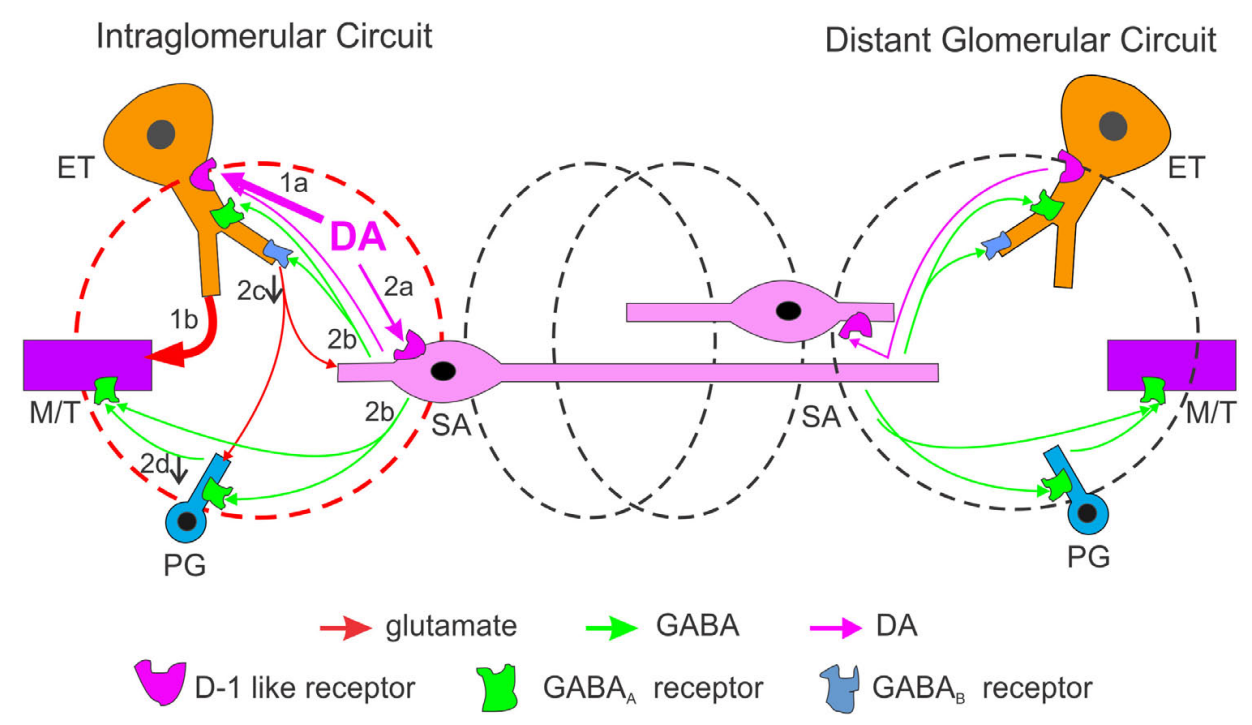

FIGURE 9 | Schematic summarizing DA regulation of OB output through intra- and inter-glomerular circuits. In the activated glomerulus (left, red), the DA net effect on output neurons is excitatory. DA directly excites two glomerular neuron types via D1LRs: the external tufted cells (ETCs, 1a) and short axon cells (SACs, 2a). Excitation of ETCs increases MC output (1b) while excited SACs corelease GABA and DA (2b) which lead to multiple consequences: (1) GABA activates GABA receptors on ETC apical dendrites to reduce glutamate release and suppress sEPSCs in postsynaptic periglomerular cells (PGCs) and SACs (2C); (2) GABA activates $\mathrm{GABA}_{A}$ receptors on PGCs to reduce glomerular inhibition of MCs (disinhibition) leading to further increase in MC output (2d); (3) GABA also inhibits MCs directly, but this inhibition is surpassed by the ETC-MC pathway-derived excitation. In the neighboring or distal glomeruli (black), output neurons are inhibited mainly by GABA-mediated interglomerular action. Thus, DA enhances the system's sensitivity through a mechanism similar to center-surround inhibition.

application (Figure 7C middle blown-up traces). After bath application of the glutamatergic receptor blockers NBQX and APV, sLLDs disappeared and glomerular application of DA showed no effect on the holding current (Figure 7C bottom trace), indicating that DA does not affect $\mathrm{MC}$ intrinsic properties and the DA-induced inward current is mediated by glutamate from ETCs. Consistently, further experiments showed that DA effects on MCs were completely blocked by SKF83566 (Figures 7D,E), indicating that they are due to activation of D1LRs in ETCs (Liu et al., 2013). In seven MCs, the average amplitude of the first and second dose of DA-induced inward current was $96.8 \pm 8.7 \mathrm{pA}$ and $91.9 \pm 8.9 \mathrm{pA}$, respectively (Figure 7D). The sLLD frequency in seven MCs was increased from $0.48 \pm 0.08 \mathrm{~Hz}$ in control to $0.83 \pm 0.08 \mathrm{~Hz}$ by the first dose of DA but resumed to $0.49 \pm 0.08 \mathrm{~Hz}$ after DA washout. The second dose of DA elevated this frequency to $0.78 \pm 0.07 \mathrm{~Hz}$ ( $p<0.05$ compared to washout of first dose of DA) followed by a recovery $(0.49 \pm 0.08 \mathrm{~Hz})$. In sum, these voltage clamp data showed that glomerular application of DA produced an excitatory net effect on MCs via D1LRs.

This conclusion led us to predict that DA enhance MC output to downstream centers. To test this, we repeated the prior experiments in current clamp (Figures 8A,B). Again, off-target glomerular application of DA did not evoke significant effect on MC membrane potential or sLLD frequency (Figure $8 \mathrm{C}$ top traces and Figure $\mathbf{8 E}$ ) while on-target DA reversibly and reliably evoked spike responses in MCs (Figure 8C middle traces and Figure 8C). Consistent with voltage clamp results, after treatment of OB slices with SKF83566 for $10 \mathrm{~min}$, on-target DA neither elicited spike response nor affected sLLDs (Figures $\mathbf{8 C}, \mathbf{E}$ ) whereas DA no longer showed any effect in the presence of NBQX and APV to completely block sLLDs (Figure 8C bottom trace). The first dose of DA increased the average instant frequency of spikes in $10 \mathrm{MCs}$ from $4.8 \pm 2.4 \mathrm{~Hz}$ to $32.2 \pm 3.8 \mathrm{~Hz}(p<0.0001)$ followed by a complete recovery $(4.9 \pm 2.4 \mathrm{~Hz}, p=1$ compared to pre-DA) while the second dose of DA reversibly elevated it up to $30.6 \pm 4.2 \mathrm{~Hz}$ ( $p<0.0001$ compared to first DA washout). These actions were completely blocked by SKF83566 (Figure 8D).

Taken together, our results demonstrate that DA produces an excitatory net effect on MCs and consequently increases OB output to downstream olfactory centers.

\section{DISCUSSION}

We characterized DA actions on both excitatory and inhibitory transmission in the glomerular circuit followed by analysis of the underlying cellular mechanisms and DA impact on $\mathrm{OB}$ output with five major findings (Figure 9). First, DA via D1LRs suppresses sEPSCs in the ETC-driven inhibitory glomerular interneurons, contradicting with DA excitatory actions on ETCs. Second, activation of D1LRs potentiates sIPSCs in all glomerular neurons, indicating enhancement of glomerular inhibition. Consistently, glomerular application of DA elevates sIPSCs in MCs. Third, the depression of sEPSCs in inhibitory glomerular interneurons is blocked by inhibition of $\mathrm{GABA}_{\mathrm{B}}$ receptors, implying that DA increases GABA release from inhibitory glomerular interneurons to inhibit glutamate release from ETC apical dendrites. Fourth, DA selectively excites the GABAergic SACs but not PGCs via D1LRs. Finally, DA produces a net 
excitatory effect on MC output via D1LRs. This effect is mediated by glutamate, suggesting the DA excitation of ETCs surpasses the enhanced glomerular inhibition thus potentiates the $\mathrm{OB}$ output to downstream targets.

\section{DA Regulates the Excitation-Inhibition Balance in the Glomerular Circuit}

Our previous study demonstrates that endogenous DA released from SACs or exogenous DA enhances ETC output by elevating the hyperpolarization-activated cation current $\left(I_{\mathrm{h}}\right)$ (Liu et al., 2013). ETCs do not have lateral dendrites and their sole apical dendrites ramify in individual glomeruli (Pinching and Powell, 1971a; Macrides and Schneider, 1982; Hayar et al., 2004b; Antal et al., 2006). Thus, they are generally considered as local excitatory interneurons participating in the operation of glomerular circuit. ETC apical dendrites form glutamatergic dendrodendritic synapses with vast majority of PGCs and SACs (Shao et al., 2009; Kiyokage et al., 2010), and apical dendrites of MTCs (De Saint et al., 2009; Gire and Schoppa, 2009) so that the overall excitation level at the glomerular level has been thought to be controlled or coordinated by ETC activities (De Saint et al., 2009). Subsequent studies with optogenetic approaches did not reveal endogenous DA actions on other postsynaptic targets of SACs including MTCs and PGCs (Banerjee et al., 2015; Liu et al., 2016; Shao et al., 2019), suggesting that DA functions as a neurotransmitter to mediate wiring transmission only from SACs to ETCs. In this context, by activating ETCs DA should increase synaptic excitation across all neuron populations in the glomerular layer. However, DA may alternatively function as a neuromodulator to mediate volume transmission given its long-lasting small quantal release from SACs (Cragg et al., 2001; Borisovska et al., 2013). In this scenario, actions of endogenous DA released from SACs may alter synaptic activities at dendritic terminals without detectable changes in membrane current or potential at cell somata where recordings are normally made from with conventional electrophysiological approaches. In other words, DA could act on neurons other than ETCs to subtly alter the synaptic excitation-inhibition balance among distinct neuron populations. Consistently, findings of the present study support this prediction, i.e., exogenous DA excites only MTCs but not the ETC-driven inhibitory glomerular interneurons PGCs and SACs, in which DA via D1LRs decreases spontaneous excitatory synaptic activities instead. Our further analysis supports that the excitatory actions of DA on MTCs are not due to direct activation of MTCs but instead are through ETC intermediation because they are eliminated by blocking the glutamatergic receptors $\mathrm{AMPA}$ and NMDA receptors, which mediate the transmission from ETCs to MTCs (De Saint et al., 2009; Gire and Schoppa, 2009). Thus, DA differentiates the ETC-driven MTCs from ETC-driven PGCs and SACs by resetting the ETCderived excitatory synaptic input to postsynaptic targets. These findings are consistent with previous studies showing that:(1) DA activates ETCs (Liu et al., 2013) which provide excitatory feedforward to MTCs (De Saint et al., 2009; Gire and Schoppa, 2009); (2) D1LRs are broadly expressed in the OB including the glomerular layer (Coronas et al., 1997; Beaulieu and Gainetdinov,
2011). Therefore, the overall impact of DA actions is to enhance the $\mathrm{OB}$ output by enhancing the ETC-intermediated excitatory feedforward to MTCs and weakening ETC-driven excitatory synaptic input to the local GABAergic interneurons PGCs and SACs, which provide inhibitory feedforward to MTCs.

\section{Cellular Mechanisms Underlying DA Actions}

Our mechanistic analysis revealed that the DA-induced suppression of excitatory synaptic activities in ETC-driven PGCs and SACs requires activation of $\mathrm{GABA}_{B}$ receptors. Activation of $\mathrm{GABA}_{\mathrm{B}}$ receptors on OSN axon terminals inhibits glutamate releases (Nickell et al., 1994; AroniadouAnderjaska et al., 2000; Wachowiak et al., 2005; McGann, 2013) whereas $\mathrm{GABA}_{B}$ receptors are also present in $\mathrm{OB}$ glomerular neurons (Fritschy et al., 1999; Panzanelli et al., 2004). Consistent with previous work showing that $\mathrm{GABA}_{\mathrm{B}}$ receptors inhibit dendrodendritic transmission from MTCs to granule cells in the OB (Isaacson and Vitten, 2003), our finding suggests that GABA released from local interneurons in response to DA activates $\mathrm{GABA}_{\mathrm{B}}$ receptors on ETC apical dendrites to reduce glutamate release causing suppression of excitatory synaptic activities in postsynaptic PGCs and SACs. This interpretation is supported by our findings that DA via D1LRs augments spontaneous inhibitory synaptic activities in all glomerular neurons and MTCs, suggesting DA increases GABA release from inhibitory interneurons in the glomerular layer. Interestingly, DA selectively excites or depolarizes SACs and increases their spontaneous firing frequency without detectable effect on PGCs. Taken together, these findings lead us to conclude that DA excites SACs, which release GABA to activate $G_{A B A}$ receptors on ETC apical dendrites and reduce glutamate release causing suppression of spontaneous excitatory synaptic activities in PGCs and SACs (Figure 9). Recent studies showed that another type of inhibitory neurons termed deep short axon cells (dSACs) have processes extending into the glomerular layer (Eyre et al., 2008; Burton et al., 2017). Future work needs to examine DA effect on dSACs to see if this population of interneuron are also subject to DAergic modulation.

\section{Functional Implications}

Similar to other brain regions like the striatum, activation of D1LR or D2LRs produces opposite effects on olfactory processing (Yue et al., 2004). In contrast to activation of D2LRs that generally produces inhibitory effects on odor discrimination and transmission of the first synapse from OSNs to postsynaptic targets in the OB (Nickell et al., 1991; Aroniadou-Anderjaska et al., 2000; Ennis et al., 2001; Yue et al., 2004; Tillerson et al., 2006; Escanilla et al., 2009; McGann, 2013), activation of D1LRs improves odor detection and discrimination (Doty et al., 1998; Yue et al., 2004). Consistently, our findings in the present study show that overall net effect of DA on $\mathrm{OB}$ output is the D1LR-mediated excitation, which depolarizes the output neurons MTCs to bring their resting membrane potential closer to the threshold of action potential generation, meanwhile, activation 
of D1LRs selectively excites SACs. Since SACs have long processes connecting multiple glomeruli (Kosaka and Kosaka, 2008; Kiyokage et al., 2010), DA activation of SACs is speculated to extend inhibition to ETCs (Liu et al., 2013; Whitesell et al., 2013) and output neurons (Banerjee et al., 2015; Liu et al., 2016) in neighboring or distal glomeruli. Thus, we propose that activation of D1LRs is to produce a center-surrounding effect thus enhances $\mathrm{OB}$ output from the activated glomeruli to facilitate odor detection and discrimination. Further work by knocking out D1LRs or chemogenetic inhibition of D1LRs in the $\mathrm{OB}$ would provide insight into this speculation.

\section{DATA AVAILABILITY STATEMENT}

All datasets generated for this study are included in the manuscript.

\section{REFERENCES}

Antal, M., Eyre, M., Finklea, B., and Nusser, Z. (2006). External tufted cells in the main olfactory bulb form two distinct subpopulations. Eur. J. Neurosci. 24, 1124-1136. doi: 10.1111/j.1460-9568.2006.04988.x

Aroniadou-Anderjaska, V., Zhou, F. M., Priest, C. A., Ennis, M., and Shipley, M. T. (2000). Tonic and synaptically evoked presynaptic inhibition of sensory input to the rat olfactory bulb via GABA(B) heteroreceptors. J. Neurophysiol. 84, 1194-1203. doi: 10.1152/jn.2000.84.3.1194

Banerjee, A., Marbach, F., Anselmi, F., Koh, M. S., Davis, M. B., Garcia da Silva, P., et al. (2015). An Interglomerular circuit gates glomerular output and implements gain control in the mouse olfactory bulb. Neuron 87, 193-207. doi: 10.1016/j.neuron.2015.06.019

Beaulieu, J. M., and Gainetdinov, R. R. (2011). The physiology, signaling, and pharmacology of dopamine receptors. Pharmacol. Rev. 63, 182-217. doi: 10 . 1124/pr.110.002642

Berkowicz, D. A., and Trombley, P. Q. (2000). Dopaminergic modulation at the olfactory nerve synapse. Brain Res. 855, 90-99. doi: 10.1016/s0006-8993(99) 02342-2

Bjorklund, A., and Dunnett, S. B. (2007a). Dopamine neuron systems in the brain: an update. Trends Neurosci. 30, 194-202. doi: 10.1016/j.tins.2007.03.006

Bjorklund, A., and Dunnett, S. B. (2007b). Fifty years of dopamine research. Trends Neurosci. 30, 185-187. doi: 10.1016/j.tins.2007.03.004

Bonzano, S., Bovetti, S., Gendusa, C., Peretto, P., and De Marchis, S. (2016). Adult born olfactory bulb dopaminergic interneurons: molecular determinants and experience-dependent plasticity. Front. Neurosci. 10:189.

Borisovska, M., Bensen, A. L., Chong, G., and Westbrook, G. L. (2013). Distinct modes of dopamine and GABA release in a dual transmitter neuron. J. Neurosci. 33, 1790-1796. doi: 10.1523/jneurosci.4342-12.2013

Burton, S. D. (2017). Inhibitory circuits of the mammalian main olfactory bulb. J. Neurophysiol. 118, 2034-2051. doi: 10.1152/jn.00109.2017

Burton, S. D., LaRocca, G., Liu, A., Cheetham, C. E., and Urban, N. N. (2017). Olfactory bulb deep short-axon cells mediate widespread inhibition of tufted cell apical dendrites. J. Neurosci. 37, 1117-1138. doi: 10.1523/jneurosci.288016.2016

Cave, J. W., and Baker, H. (2009). Dopamine systems in the forebrain. Adv. Exp. Med. Biol. 651, 15-35. doi: 10.1007/978-1-4419-0322-8_2

Coronas, V., Srivastava, L. K., Liang, J. J., Jourdan, F., and Moyse, E. (1997). Identification and localization of dopamine receptor subtypes in rat olfactory mucosa and bulb: a combined in situ hybridization and ligand binding radioautographic approach. J. Chem. Neuroanat. 12, 243-257. doi: 10.1016/ s0891-0618(97)00215-9

Cragg, S. J., Nicholson, C., Kume-Kick, J., Tao, L., and Rice, M. E. (2001). Dopamine-mediated volume transmission in midbrain is regulated by distinct extracellular geometry and uptake. J. Neurophysiol. 85, 1761-1771. doi: 10. 1152/jn.2001.85.4.1761

\section{ETHICS STATEMENT}

The animal study was reviewed and approved by Howard University Animal Care and Use Committees.

\section{AUTHOR CONTRIBUTIONS}

SL planned and performed the experiments, analyzed the data, performed the statistical analysis, interpreted the results, wrote the manuscript, and approved the content.

\section{FUNDING}

This project was supported by the National Institutes of Health, NIDCDR01DC014447 to SL.

Dahlstroem, A., and Fuxe, K. (1964). Evidence for the existence of monoaminecontaining neurons in the central nervous system. I. Demonstration of monoamines in the cell bodies of brain stem neurons. Acta Physiol. Scand. Suppl, 232, 231-255.

De Saint, J. D., Hirnet, D., Westbrook, G. L., and Charpak, S. (2009). External tufted cells drive the output of olfactory bulb glomeruli. J. Neurosci. 29, 2043-2052. doi: 10.1523/jneurosci.5317-08.2009

Doty, R. L., Li, C., Bagla, R., Huang, W., Pfeiffer, C., Brosvic, G. M., et al. (1998). SKF 38393 enhances odor detection performance. Psychopharmacology 136, 75-82. doi: 10.1007/s002130050541

Ennis, M., Zhou, F. M., Ciombor, K. J., Aroniadou-Anderjaska, V., Hayar, A., Borrelli, E., et al. (2001). Dopamine D2 receptor-mediated presynaptic inhibition of olfactory nerve terminals. J. Neurophysiol. 86, 2986-2997. doi: 10.1152/jn.2001.86.6.2986

Escanilla, O., Yuhas, C., Marzan, D., and Linster, C. (2009). Dopaminergic modulation of olfactory bulb processing affects odor discrimination learning in rats. Behav. Neurosci. 123, 828-833. doi: 10.1037/a0015855

Eyre, M. D., Antal, M., and Nusser, Z. (2008). Distinct deep short-axon cell subtypes of the main olfactory bulb provide novel intrabulbar and extrabulbar GABAergic connections. J. Neurosci. 28, 8217-8229. doi: 10.1523/jneurosci. 2490-08.2008

Fritschy, J. M., Meskenaite, V., Weinmann, O., Honer, M., Benke, D., and Mohler, H. (1999). GABAB-receptor splice variants GBla and GB1b in rat brain: developmental regulation, cellular distribution and extrasynaptic localization. Eur. J. Neurosci. 11, 761-768. doi: 10.1046/j.1460-9568.1999. 00481.x

Gire, D. H., and Schoppa, N. E. (2009). Control of on/off glomerular signaling by a local GABAergic microcircuit in the olfactory bulb. J. Neurosci. 29, 13454-13464. doi: 10.1523/jneurosci.2368-09.2009

Guthrie, K. M., Pullara, J. M., Marshall, J. F., and Leon, M. (1991). Olfactory deprivation increases dopamine D2 receptor density in the rat olfactory bulb. Synapse 8, 61-70. doi: 10.1002/syn.890080109

Gutierrez-Mecinas, M., Crespo, C., Blasco-Ibanez, J. M., Gracia-Llanes, F. J., Marques-Mari, A. I., Nacher, J., et al. (2005). Distribution of D2 dopamine receptor in the olfactory glomeruli of the rat olfactory bulb. Eur. J. Neurosci. 22, 1357-1367. doi: 10.1111/j.1460-9568.2005.04328.x

Hayar, A., Karnup, S., Ennis, M., and Shipley, M. T. (2004a). External tufted cells: a major excitatory element that coordinates glomerular activity. J. Neurosci. 24, 6676-6685. doi: 10.1523/jneurosci.1367-04.2004

Hayar, A., Karnup, S., Shipley, M. T., and Ennis, M. (2004b). Olfactory bulb glomeruli: external tufted cells intrinsically burst at theta frequency and are entrained by patterned olfactory input. J. Neurosci. 24, 1190-1199. doi: 10.1523/ jneurosci.4714-03.2004

Hsia, A. Y., Vincent, J. D., and Lledo, P. M. (1999). Dopamine depresses synaptic inputs into the olfactory bulb. J. Neurophysiol. 82, 1082-1085. doi: 10.1152/jn. 1999.82.2.1082 
Isaacson, J. S., and Vitten, H. (2003). GABA(B) receptors inhibit dendrodendritic transmission in the rat olfactory bulb. J. Neurosci. 23, 2032-2039. doi: 10.1523/ jneurosci.23-06-02032.2003

Karpuk, N., and Hayar, A. (2008). Activation of postsynaptic GABAB receptors modulates the bursting pattern and synaptic activity of olfactory bulb juxtaglomerular neurons. J. Neurophysiol. 99, 308-319. doi: 10.1152/jn.01086. 2007

Kiyokage, E., Pan, Y. Z., Shao, Z., Kobayashi, K., Szabo, G., Yanagawa, Y., et al. (2010). Molecular identity of periglomerular and short axon cells. J. Neurosci. 30, 1185-1196. doi: 10.1523/jneurosci.3497-09.2010

Klein, M. O., Battagello, D. S., Cardoso, A. R., Hauser, D. N., Bittencourt, J. C., and Correa, R. G. (2019). Dopamine: functions, signaling, and association with neurological diseases. Cell Mol. Neurobiol. 39, 31-59. doi: 10.1007/s10571-0180632-3

Kosaka, T., and Kosaka, K. (2008). Tyrosine hydroxylase-positive GABAergic juxtaglomerular neurons are the main source of the interglomerular connections in the mouse main olfactory bulb. Neurosci. Res. 60, 349-354. doi: 10.1016/j.neures.2007.11.012

Lazarini, F., Gabellec, M. M., Moigneu, C., de Chaumont, F., Olivo-Marin, J. C., and Lledo, P. M. (2014). Adult neurogenesis restores dopaminergic neuronal loss in the olfactory bulb. J. Neurosci. 34, 14430-14442. doi: 10.1523/jneurosci. 5366- 13.2014

Levey, A. I., Hersch, S. M., Rye, D. B., Sunahara, R. K., Niznik, H. B., Kitt, C. A., et al. (1993). Localization of D1 and D2 dopamine receptors in brain with subtype-specific antibodies. Proc. Natl. Acad. Sci. U.S.A. 90, 8861-8865. doi: 10.1073/pnas.90.19.8861

Liu, S., Plachez, C., Shao, Z., Puche, A., and Shipley, M. T. (2013). Olfactory bulb short axon cell release of GABA and dopamine produces a temporally biphasic inhibition-excitation response in external tufted cells. J. Neurosci. 33, 2916-2926. doi: 10.1523/jneurosci.3607-12.2013

Liu, S., Puche, A. C., and Shipley, M. T. (2016). The interglomerular circuit potently inhibits olfactory bulb output neurons by both direct and indirect pathways. J. Neurosci. 36, 9604-9617. doi: 10.1523/jneurosci.1763-16.2016

Lledo, P. M., Gheusi, G., and Vincent, J. D. (2005). Information processing in the mammalian olfactory system. Physiol. Rev. 85, 281-317. doi: 10.1152/physrev. 00008.2004

Lopez-Bendito, G., Sturgess, K., Erdelyi, F., Szabo, G., Molnar, Z., and Paulsen, O. (2004). Preferential origin and layer destination of GAD65-GFP cortical interneurons. Cereb. Cortex 14, 1122-1133. doi: 10.1093/cercor/bhh072

Macrides, F., and Schneider, S. P. (1982). Laminar organization of mitral and tufted cells in the main olfactory bulb of the adult hamster. J. Comp. Neurol. 208 , 419-430. doi: 10.1002/cne.902080410

Maher, B. J., and Westbrook, G. L. (2008). Co-transmission of dopamine and GABA in periglomerular cells. J. Neurophysiol. 99, 1559-1564. doi: 10.1152/ jn.00636.2007

Matsushita, N., Okada, H., Yasoshima, Y., Takahashi, K., Kiuchi, K., and Kobayashi, K. (2002). Dynamics of tyrosine hydroxylase promoter activity during midbrain dopaminergic neuron development. J. Neurochem. 82, 295-304. doi: 10.1046/j. 1471-4159.2002.00972.x

McGann, J. P. (2013). Presynaptic inhibition of olfactory sensory neurons: new mechanisms and potential functions. Chem. Sens. 38, 459-474.

Nagayama, S., Homma, R., and Imamura, F. (2014). Neuronal organization of olfactory bulb circuits. Front. Neural. Circ. 8:98.

Nickell, W. T., Behbehani, M. M., and Shipley, M. T. (1994). Evidence for GABABmediated inhibition of transmission from the olfactory nerve to mitral cells in the rat olfactory bulb. Brain Res. Bull. 35, 119-123. doi: 10.1016/0361-9230(94) 90091-4

Nickell, W. T., Norman, A. B., Wyatt, L. M., and Shipley, M. T. (1991). Olfactory bulb DA receptors may be located on terminals of the olfactory nerve. Neuroreport 2, 9-12. doi: 10.1097/00001756-199101000-00002

Panzanelli, P., Lopez-Bendito, G., Lujan, R., and Sassoe-Pognetto, M. (2004). Localization and developmental expression of GABA(B) receptors in the rat olfactory bulb. J. Neurocytol. 33, 87-99. doi: 10.1023/b:neur.0000029650.28 943.b2

Parrish-Aungst, S., Kiyokage, E., Szabo, G., Yanagawa, Y., Shipley, M. T., and Puche, A. C. (2011). Sensory experience selectively regulates transmitter synthesis enzymes in interglomerular circuits. Brain Res. 1382, 70-76. doi: 10.1016/j.brainres.2011.01.068

Pignatelli, A., and Belluzzi, O. (2017). Dopaminergic neurones in the main olfactory bulb: an overview from an electrophysiological perspective. Front. Neuroanat. 11:7.

Pinching, A. J., and Powell, T. P. (1971a). The neuron types of the glomerular layer of the olfactory bulb. J. Cell Sci. 9, 305-345.

Pinching, A. J., and Powell, T. P. (1971b). The neuropil of the periglomerular region of the olfactory bulb. J. Cell Sci. 9, 379-409.

Romon, Y., and Cajal, S. (1909). Histologie du Systeİme Nerveux de l'homme \& des Verteibreis. Paris: Maloine.

Serguera, C., Triaca, V., Kelly-Barrett, J., Banchaabouchi, M. A., and Minichiello, L. (2008). Increased dopamine after mating impairs olfaction and prevents odor interference with pregnancy. Nat. Neurosci. 11, 949-956. doi: 10.1038/nn. 2154

Shao, Z., Puche, A. C., Kiyokage, E., Szabo, G., and Shipley, M. T. (2009). Two GABAergic intraglomerular circuits differentially regulate tonic and phasic presynaptic inhibition of olfactory nerve terminals. J. Neurophysiol. 101, 19882001. doi: 10.1152/jn.91116.2008

Shao, Z., Puche, A. C., Liu, S., and Shipley, M. T. (2012). Intraglomerular inhibition shapes the strength and temporal structure of glomerular output. J. Neurophysiol. 108, 782-793. doi: 10.1152/jn.00119.2012

Shao, Z., Liu, S., Zhou, F., Puche, A. C., and Shipley, M. T. (2019). Reciprocal inhibitory glomerular circuits contribute to excitation-inhibition balance in the mouse olfactory bulb. eNeuro 6:ENEURO.0048-19. doi: 10.1523/ENEURO. 0048-19.2019

Taylor, T. N., Caudle, W. M., Shepherd, K. R., Noorian, A., Jackson, C. R., Iuvone, P. M., et al. (2009). Nonmotor symptoms of Parkinson's disease revealed in an animal model with reduced monoamine storage capacity. J. Neurosci. 29, 8103-8113. doi: 10.1523/jneurosci.1495-09.2009

Tillerson, J. L., Caudle, W. M., Parent, J. M., Gong, C., Schallert, T., and Miller, G. W. (2006). Olfactory discrimination deficits in mice lacking the dopamine transporter or the D2 dopamine receptor. Behav. Brain Res. 172, 97-105. doi: 10.1016/j.bbr.2006.04.025

Vaaga, C. E., Yorgason, J. T., Williams, J. T., and Westbrook, G. L. (2017). Presynaptic gain control by endogenous cotransmission of dopamine and GABA in the olfactory bulb. J. Neurophysiol. 117, 1163-1170. doi: 10.1152/jn. 00694.2016

Wachowiak, M., McGann, J. P., Heyward, P. M., Shao, Z., Puche, A. C., and Shipley, M. T. (2005). Inhibition [corrected] of olfactory receptor neuron input to olfactory bulb glomeruli mediated by suppression of presynaptic calcium influx. J. Neurophysiol. 94, 2700-2712. doi: 10.1152/jn.00286.2005

Whitesell, J. D., Sorensen, K. A., Jarvie, B. C., Hentges, S. T., and Schoppa, N. E. (2013). Interglomerular lateral inhibition targeted on external tufted cells in the olfactory bulb. J. Neurosci. 33, 1552-1563. doi: 10.1523/jneurosci.3410-12.2013

Wilson, R. I., and Mainen, Z. F. (2006). Early events in olfactory processing. Annu. Rev. Neurosci. 29, 163-201. doi: 10.1146/annurev.neuro.29.051605. 112950

Yu, Q., Liu, Y. Z., Zhu, Y. B., Wang, Y. Y., Li, Q., and Yin, D. M. (2019). Genetic labeling reveals temporal and spatial expression pattern of D2 dopamine receptor in rat forebrain. Brain Struct. Funct. 224, 1035-1049. doi: 10.1007/ s00429-018-01824-2

Yue, E. L., Cleland, T. A., Pavlis, M., and Linster, C. (2004). Opposing effects of D1 and D2 receptor activation on odor discrimination learning. Behav. Neurosci. 118, 184-190. doi: 10.1037/0735-7044.118.1.184

Conflict of Interest: The author declares that the research was conducted in the absence of any commercial or financial relationships that could be construed as a potential conflict of interest.

Copyright (c) 2020 Liu. This is an open-access article distributed under the terms of the Creative Commons Attribution License (CC BY). The use, distribution or reproduction in other forums is permitted, provided the original author(s) and the copyright owner(s) are credited and that the original publication in this journal is cited, in accordance with accepted academic practice. No use, distribution or reproduction is permitted which does not comply with these terms. 